\title{
Homogenization of processes in nonlinear visco-elastic composites
}

\author{
Augusto Visintin
}

\begin{abstract}
The constitutive behaviour of a multiaxial visco-elastic material is here represented by the nonlinear relation

$$
\varepsilon-A(x): \int_{0}^{t} \sigma(x, \tau) d \tau \in \alpha(\sigma, x),
$$

which generalizes the classical Maxwell model of visco-elasticity of fluid type. Here $\alpha(\cdot, x)$ is a (possibly multivalued) maximal monotone mapping, $\sigma$ is the stress tensor, $\varepsilon$ is the linearized strain tensor, and $A(x)$ is a positive-definite fourth-order tensor. The above inclusion is here coupled with the quasi-static force-balance law, $-\operatorname{div} \sigma=\vec{f}$. Existence and uniqueness of the weak solution are proved for a boundary-value problem.

Convergence to a two-scale problem is then derived for a composite material, in which the functions $\alpha$ and $A$ periodically oscillate in space on a short length-scale. It is proved that the coarse-scale averages of stress and strain solve a single-scale homogenized problem, and that conversely any solution of this problem can be represented in that way. The homogenized constitutive relation is represented by the minimization of a time-integrated functional, and is rather different from the above constitutive law. These results are also retrieved via De Giorgi's notion of $\Gamma$-convergence. These conclusions are at variance with the outcome of so-called analogical models, that rest on an (apparently unjustified) mean-field-type hypothesis.
\end{abstract}

Mathematics Subject Classification (2010): 35B27 (primary); 49J40, 73E50, 74Q (secondary).

\section{Introduction}

In this work we deal with processes in nonlinear visco-elastic composite materials of fluid type, and illustrate a method of homogenization based on the use of two length-scales.

A nonlinear visco-elastic law. Throughout this paper we make the assumption of infinitesimal displacements, and use the linearized strain-tensor $\varepsilon$. Denoting

Received September 17, 2008; accepted in revised form May 26, 2010. 
the stress-tensor by $\sigma$, we represent a visco-elastic behaviour of fluid type via a constitutive relation of the form

$$
\varepsilon-A(x): \int_{0}^{t} \sigma(x, \tau) d \tau \in \alpha(\sigma, x)
$$

that is, the differential equation $\partial \varepsilon / \partial t+A(x): \sigma=\partial \xi / \partial t$ for some $\xi \in \alpha(\sigma, x)$, coupled with the initial condition $\varepsilon(0)=\xi(0) \in \alpha(\sigma(0), x)$. Here $\alpha(\cdot, x)$ is a (possibly multivalued) maximal monotone mapping in the space of symmetric secondorder tensors, $A(x)$ is a positive-semidefinite fourth-order tensor, and ":" denotes the contraction over two indices. (1.1) is of the form $\varepsilon \in \gamma(\sigma, x)$ for a.e. $x$, with $\gamma(\cdot, x)$ a maximal monotone operator in the space $L^{2}(0, T)_{S}^{9}$ (of tensor-valued mappings, see Section 2). This inclusion is tantamount to the variational inequality

$\left(\varepsilon-A(x): \int_{0}^{t} \sigma(x, \tau) d \tau-z\right):(\sigma-v) \geq 0 \quad \forall(v, z)$ such that $z \in \alpha(v, x)$.

This accounts for a fluid type visco-elastic behaviour, for here $\varepsilon$ may indefinitely grow under constant stress. For a linear $\alpha(\cdot, x)$ we thus retrieve the classical Maxwell model of visco-elasticity, see $e . g$. $[56,76]$.

A two-scale homogenization program. In Section 2 we briefly discuss the rheological model, and outline the Fitzpatrick representation of maximal monotone operators, see [42], which plays a key role in this work. Afterwards we formulate a number of models along the following lines:

(i) Model of a macroscopically inhomogeneous but mesoscopically homogeneous material

In Section 3 we couple the relation (1.1) with the quasi-static equilibrium equation in a domain $\Omega$ of $\mathbf{R}^{3}$ in a time interval $] 0, T$,

$$
\left.-\nabla \cdot \sigma=\vec{f}_{1} \quad \text { in } \Omega \times\right] 0, T[(\nabla \cdot:=\operatorname{div}),
$$

and with appropriate boundary-conditions. We formulate a boundary-value problem, $P$, in the framework of Sobolev spaces, and via standard techniques we prove that it has one and only one solution $(\sigma, \varepsilon)$.

(ii) Model of a mesoscopically inhomogeneous material

We then deal with a composite material, that by a classical procedure we replace by a family of materials parameterized by the length-scale $\eta<<1$, in which the constitutive data $A$ and $\alpha$ periodically depend on $x / \eta$. The corresponding problem, $P_{\eta}$, has the same structure as $P$, and thus has one and only one solution $\left(\sigma_{\eta}, \varepsilon_{\eta}\right)$ for any $\eta$. We also show that this family is uniformly bounded in appropriate function spaces.

(iii) Two-scale model

In Section 4 we introduce a fine-scale variable $y=x / \eta$, that we may let range through a reference set $Y:=\left[0,1\left[{ }^{3}\right.\right.$ because of the periodicity. We formulate a 
two-scale problem, $P_{2}$, in which both the coarse- and fine-scale variables $x$ and $y$ occur, and (1.1) is replaced by the two-scale constitutive law

$$
\left.\varepsilon(x, y, t)-A(y, t): \int_{0}^{t} \sigma(x, y, \tau) d \tau \in \alpha(\sigma(x, y, t), y) \quad \text { for }(x, y, t) \in \Omega \times Y \times\right] 0, T[.
$$

We then show that as $\eta \rightarrow 0$ a suitable sequence of solutions of $P_{\eta}$ two-scale converges (in the sense of Nguetseng [ [73]]) to a solution of problem $P_{2}$.

(iv) Scale-transformations of the constitutive law

This and the next two steps are the main concern of the present work. In view of the homogenization of the two-scale problem, first in Section 5 we deal with the homogenization of the constitutive relation. For this purpose we uncouple the twoscale relation (1.4) from the force-balance equation (1.3), and drop the variable $x$. From this cell problem we derive a coarse-scale relation of the form

$$
\Lambda(\hat{\sigma}, \hat{\varepsilon})=\int_{0}^{T} \hat{\sigma}: \hat{\varepsilon} d t
$$

for the average fields $\hat{\sigma}(t):=\int_{Y} \sigma(y, t) d y$ and $\hat{\varepsilon}(t):=\int_{Y} \varepsilon(y, t) d y$ (so-called upscaling), Here $\Lambda$ is the infimum of a family of time-integral functionals, $c f$. (5.14), and is convex, lower semicontinuous and coercive. We also show that, conversely, all functions $\hat{\sigma}(t)$ and $\hat{\varepsilon}(t)$ that satisfy this coarse-scale relation can be represented as averages of suitable fields $\sigma(y, t)$ and $\varepsilon(y, t)$ that fulfill the two-scale law (1.4) (so-called downscaling).

On the basis of a general result, see [90], we then express the relation (1.5) in the form

$$
\hat{\varepsilon} \in \beta(\hat{\sigma}) \quad \text { a.e. in }] 0, T[\text {. }
$$

Here $\beta$ is a maximal monotone operator in the space $L^{2}(0, T)_{S}^{9}$, hence also in $L^{2}(\Omega \times] 0, T[)_{s}^{9}$ by an obvious identification. Although, as we saw, the inhomogeneous constitutive law may be presented by a first-order ordinary differential equation, this fails for the homogenized relation. This has a quite different formulation, that may exhibit a long memory - and it may be expected that so it does.

(v) Single-scale homogenization of the two-scale problem (upscaling)

In Section 6 we extend the above direct and inverse scale-transformations to the boundary-value problem. We derive a single-scale problem, $P_{1}$, from the two-scale problem $P_{2}$, and show that the $Y$-average of any solution of $P_{2}$ solves $P_{1}$. So as $\eta \rightarrow 0$ a sequence of solutions of $P_{\eta}$ single-scale converges to a solution of $P_{1}$, that thus represents the homogenized problem. This final formulation consists of an initial-boundary-value problem for the system of (1.6) coupled with the balance law (1.3). 
(vi) Inversion of the scale-transformation (downscaling)

Conversely, we show that any solution of the effective problem $P_{1}$ is the $Y$-average of a solution of problem $P_{2}$. With a terminology mutuated from [40], this inverse scale-trasformation defines a reconstruction operator, whereas the $Y$-average is a compression operator,

(vii) Interpretation by $\Gamma$-convergence

Thanks to the variational formulation first of the monotone relation and then of the whole problem, we are also able to retrieve the homogenization result via De Giorgi's notion of $\Gamma$-convergence. Concerning this issue, it may be noticed that the representation of the $\Gamma$-limit is provided by the above construction.

These conclusions are at variance with the outcome of so-called analogical models, that rest upon an (apparently unjustified) mean-field-type hypothesis.

Remarks. We have thus replaced the passage to the limit in Problem $3.1_{\eta}$ by a two-step procedure: first the derivation of the two-scale formulation, and then the upscaling. Although a priori some loss of information might be expected because of the integration with respect to the fine-scale variable $y$, it turns out that the twoand single-scale problems convey equivalent information, albeit in different form. The single-scale formulation is obviously more economical, in that it deals with a more restricted number of independent variables, and is more prone to numerical simulations. It may also be noticed that this single-scale constitutive relation is at variance with customary models of visco-elasticity.

In this work we assume the multivalued mapping $\alpha(\cdot, x)$ to be maximal monotone, without any hypothesis of cyclical monotonicity. By means of the theory pioneered by Fitzpatrick [42], we are able to represent the maximal monotone relation (1.1) in variational form:

$$
\text { Find such }(\sigma, \varepsilon) \text { that } J(\sigma, \varepsilon)=\inf J(=0) .
$$

In the next section we shall define the functional $J$, and briefly illustrate this derivation.

We are able to express the macroscopic behaviour of our system just in terms of the coarse-scale fields $\hat{\sigma}$ and $\hat{\varepsilon}$. After a classical result of Marcellini [60], an analogous conclusion is already known to hold for (stationary) minimization principles; see e.g. [17, 18,24,30,35]. On the other hand, the present homogenization result applies to several evolutionary variational inequalities, that are not included in that class.

This result rests on certain orthogonality properties, see the mutually orthogonal spaces $W$ and $Z$ that are defined in (5.4). This is reminiscent of the analytical structure that underlies Murat and Tartar's theory of compensated compactness, $c f$. e.g. $[68,69,82,83]$. Similar orthogonality and convexity properties also occur in other physical phenomena, e.g. in electromagnetism and heat conduction, see [88].

Literature. Elasticity and viscosity were dealt with in many monographs, see e.g. $[1,6,28,38,43,48-50,55-57,71,77]$. 
A model of visco-elasticity was coupled with the equation of continuum dynamics in $[15,16,57]$; see also e.g. $[44,45,79]$ for the associated homogenization. It is well-known that nonlinear elasticity may be represented within the finite-strain theory, see e.g. $[13,28,34]$ : this might then be expected to provide an appropriate framework for visco-elasticity, too, but no result in this direction is known to this author.

Homogenization was addressed by a large literature, starting with the seminal works of Babuška [10], Bensoussan, Lions and Papanicolaou [14], De Giorgi and Spagnolo [37], Sanchez-Palencia [79], Tartar [82], and others. See e.g. [5, 12, 17, $18,25,33,35,52,54,66,69,74,75]$.

The notion of two-scale convergence is due to Nguetseng [73] (see also the seminal paper [7]), was further developed by Allaire [4], and then applied in a large and increasing number of papers. See also the reformulation of Cioranescu, Damlamian and Griso [31,32] via periodic unfolding, and the review paper [59].

Two-scale convergence was applied to the homogenization of the (stationary) Hencky model of elasto-plasticity in [26], and of quasi-stationary processes for a wide class of inelastic composite materials in $[3,72]$. The two-scale homogenization of quasi-static elasto-plastic processes with strain-hardening was dealt with in [65], via what is known as the energetic approach to rate-independent evolution. In [11] two-scale convergence was also applied to the study of $\Gamma$-convergence (for the latter, see e.g. $[17,18,35,36])$ for quasiconvex functionals; see also , [30].

After the seminal work of Fitzpatrick [42], the representation of monotone operators has extensively been developed by several authors, for instance Burachik, Martinez-Legaz, Svaiter, and others; see e.g. [22, 23,61-63]. See also the theory developed by Ghoussoub in [47].

The present work illustrates a method that may also be applied to a large number of variational inequalities that occur in continuum mechanics, electromagnetism and heat conduction. For instance, the homogenization of nonlinear extensions of the Maxwell and Kelvin-Voigt models of visco-elasticity was studied in this way in $[85,87]$, including the inertial term in the equation of continuum dynamics. A relation of the form

$$
\sigma-A^{-1}(x): \frac{\partial \varepsilon}{\partial t} \in \alpha^{-1}(\varepsilon, x)
$$

accounts for a visco-elastic behaviour of solid-type, for here under constant stress $\varepsilon$ remains bounded. The homogenization of non-quasi-static processes in composites of this type was dealt with in [89].

ACKNOWLEDGEMENTS. The author gratefully acknowledges several useful remarks of the anonymous reviewer. 


\section{The rheological model and Fitzpatrick representation}

In this section we outline our model of visco-elasticity, and review some elements of the Fitzpatrick theory.

First let us fix some notation. We shall mark vectors by an arrow, but use no special symbol for second- and higher-order tensors. We shall denote by $\mathbf{R}^{9}$ the linear space of $3 \times 3$ Cartesian tensors (or rather of their representation in a fixed Cartesian reference system), and by $\mathbf{R}_{s}^{9}$ the linear subspace of symmetric tensors; we shall also write $L^{2}(\Omega)_{s}^{9}$ in place of $L^{2}\left(\Omega ; \mathbf{R}_{s}^{9}\right)$, and use similar notation for other spaces of tensor-valued functions. We shall denote the scalar product between two vectors (i.e., the contraction over an index) by ".", and that between two matrices (i.e., the contraction over two indices) by “:”. Thus

$$
\begin{aligned}
& u: v=\sum_{i, j=1}^{3} u_{i j} v_{i j}, \quad(B: v)_{i j}=\sum_{k, \ell=1}^{3} B_{i j k \ell} v_{k \ell}, \\
& u: B: v=\sum_{i, j, k, \ell=1}^{3} u_{i j} B_{i j k \ell} v_{k \ell} \quad \forall u, v \in \mathbf{R}^{9}, \forall B \in \mathbf{R}^{3^{4}}, \\
& (A: B)_{i j m n}=\sum_{k, \ell=1}^{3} A_{i j k \ell} B_{k \ell m n} \quad \forall A, B \in \mathbf{R}^{3^{4}} .
\end{aligned}
$$

We define the spheric and deviatoric components of any $v \in \mathbf{R}^{9}$ :

$$
v_{(s)}:=\frac{1}{3} \sum_{i=1}^{3} v_{i i} I \quad\left(I:=\left\{\delta_{i j}\right\}: 3 \times 3 \text {-identity tensor }\right), \quad v_{(d)}:=v-v_{(s)}
$$

Rheological behaviour. We shall deal with mechanical processes in a continuum, assuming that displacements are so small that we can identify the Euler and Lagrange coordinates. We shall denote the Cauchy stress tensor by $\sigma$, the displacement field by $\vec{u}$, and use the linearized strain tensor

$$
\varepsilon_{i j}:=\left(\nabla^{s} \vec{u}\right)_{i j}=\frac{1}{2}\left(\frac{\partial u_{i}}{\partial x_{j}}+\frac{\partial u_{j}}{\partial x_{i}}\right) \quad \text { for } i, j=1,2,3 .
$$

In the literature a number of constitutive relations has been formulated in terms of $\sigma$, $\varepsilon$ and $\dot{\varepsilon}$ (by the dot we denote the partial time-derivative), cf. e.g. $[1,2,43,49,56,76]$. Here we just consider two basic behaviours:

(i) Linear viscosity

This may be represented by a law of the form

$$
\dot{\varepsilon}=A: \sigma
$$


here $A$ is a symmetric and positive-semidefinite tensor of $\mathbf{R}^{3^{4}}$, and $\sum_{i=1}^{3} \dot{\varepsilon}_{i i}=0$ :

$$
\begin{aligned}
& A_{i j k \ell}=A_{j i k \ell} \quad \forall i, j, k, \ell, \quad \sum_{i=1,2,3} A_{i i k \ell}=0 \quad \forall k, \ell, \\
& A_{i j k \ell} v_{i j} v_{k \ell} \geq 0 \quad \forall v \in \mathbf{R}_{s}^{9} .
\end{aligned}
$$

(ii) Nonlinear elasticity

We represent nonlinear elasticity by the relation

$$
\varepsilon \in \alpha(\sigma) \text { for a maximal monotone mapping } \alpha: \mathbf{R}_{s}^{9} \rightarrow \mathcal{P}\left(\mathbf{R}_{s}^{9}\right)
$$

(by $\mathcal{P}\left(\mathbf{R}_{s}^{9}\right)$ we denote the power set). This also encompasses linear (possibly anisotropic) elasticity, viz. $\varepsilon=L: \sigma$ for some positive-definite fourth-order compliance tensor $L \in \mathbf{R}^{3^{4}}$ such that $L_{i j k \ell}=L_{j i k \ell}$ for any $i, j, k, \ell \in\{1,2,3\}$. Although the standard theory assumes that $\alpha$ admits a potential, namely, $L_{i j k \ell}=L_{k \ell i j}$ in the linear case, here we shall not need this restriction.

The relation (2.5) is expressed in terms of the linearized strain tensor, $\varepsilon$, and thus rests on the assumption of infinitesimal displacements. This relation might be regarded as an approximation of a finite-displacement constitutive law. Although such a model would be much more satisfactory from the mechanical point of view, its analysis looks rather problematic; actually, so far that theory has been developed for nonlinear elasticity just in the stationary framework.

Although the range of validity of (2.5) is necessarily confined to a neighbourhood of the origin (of $\mathbf{R}_{s}^{9}$ ), this relation might be regarded as an improvement over the linear stress-strain relation $\varepsilon=L: \sigma$, because of its greater generality. For instance, a mapping $\sigma \mapsto B(\sigma): \sigma$ is maximal monotone in a neighbourhood $V$ of the origin, whenever $B(0)$ is positive-definite and $B(\cdot)\left(\in \mathbf{R}^{9 \times 9}\right)$ is a continuously differentiable tensor-function in $V$. In fact, for any $\sigma_{1}, \sigma_{2} \in \mathbf{R}_{s}^{9}$, by the mean-value theorem there exists $\lambda \in] 0,1\left[\right.$ such that, setting $\sigma_{\lambda}=\lambda \sigma_{1}+(1-\lambda) \sigma_{2}$ and denoting by $D$ the Jacobian derivative,

$$
\begin{aligned}
& {\left[B\left(\sigma_{1}\right): \sigma_{1}-B\left(\sigma_{2}\right): \sigma_{2}\right]:\left(\sigma_{1}-\sigma_{2}\right) } \\
= & \left(\sigma_{1}-\sigma_{2}\right):\left\{D[B(\sigma): \sigma]_{\sigma=\sigma_{\lambda}}\right\}:\left(\sigma_{1}-\sigma_{2}\right) \\
= & \left(\sigma_{1}-\sigma_{2}\right):\left\{\left[D B\left(\sigma_{\lambda}\right)\right]: \sigma_{\lambda}+B\left(\sigma_{\lambda}\right)\right\}:\left(\sigma_{1}-\sigma_{2}\right) .
\end{aligned}
$$

This quantity is nonnegative whenever $\sigma_{1}$ and $\sigma_{2}$ are sufficiently close to 0 , for then the tensor $B\left(\sigma_{\lambda}\right)$ is uniformly positive-definite in $V$ and dominates

$$
\left[D B\left(\sigma_{\lambda}\right)\right]: \sigma_{\lambda}=\sum_{i, j=1}^{3}\left[\partial B\left(\sigma_{\lambda}\right) / \partial \sigma_{i j}\right]: \sigma_{\lambda i j}=\mathcal{O}(\sigma) .
$$

Further constitutive behaviours may be derived by composing the above properties via series and/or parallel arrangements. For instance, the serial combination of the above elastic and viscous elements corresponds to the relation (1.1). On 
the other hand, the parallel arrangement of the same elements is represented by the dual constitutive law (1.8). Strictly speaking, series and parallel arrangements are meaningful only in the univariate setting; however, these relations may easily be extended to multivariate models, just by assuming the uniformity either of stress or of strain, respectively; see e.g. [87]. For a linear $\alpha,(1.1)$ and (1.8) respectively account for the classical Maxwell and Kelvin-Voigt models, that are idealized representations respectively of the visco-elastic behaviour of fluid and solid materials $[1,2,43,49,55,56,64,76]$. These relations encompass the basic elements (i) and (ii) above; for instance, these are respectively retrieved from (1.1) by selecting either $\alpha=0$ or $A=0$.

The Fitzpatrick representation of maximal monotone operators. Fitzpatrick established the following result.

Theorem 2.1 ([42]). Let $B$ be a real Banach space, $\gamma$ be an operator $B \rightarrow \mathcal{P}\left(B^{\prime}\right)$, and set

$$
f_{\gamma}\left(\xi, \xi^{\prime}\right):=\sup \left\{\left\langle\xi^{\prime}, \xi_{0}\right\rangle+\left\langle\xi_{0}^{\prime}, \xi\right\rangle-\left\langle\xi_{0}^{\prime}, \xi_{0}\right\rangle: \xi_{0} \in B, \xi_{0}^{\prime} \in \gamma\left(\xi_{0}\right)\right\} \quad \forall\left(\xi, \xi^{\prime}\right) \in B \times B^{\prime} .
$$

$\gamma$ is maximal monotone if and only if

$$
\begin{array}{cc}
f_{\gamma}\left(\xi, \xi^{\prime}\right) \geq\left\langle\xi^{\prime}, \xi\right\rangle & \forall\left(\xi, \xi^{\prime}\right) \in B \times B^{\prime}, \\
f_{\gamma}\left(\xi, \xi^{\prime}\right)=\left\langle\xi^{\prime}, \xi\right\rangle \quad \Leftrightarrow \quad \xi^{\prime} \in \gamma(\xi) .
\end{array}
$$

The function $f_{\gamma}$ is convex and lower semicontinuous; nowadays it is called the Fitzpatrick function associated to the operator $\gamma$.

Coming back to our model of visco-elasticity, for any $x \in \Omega$, setting

$$
\varphi(\xi, \eta, x):=\sup _{\eta_{0} \in \alpha\left(\xi_{0}, x\right)}\left\{\eta: \xi_{0}-\eta_{0}:\left(\xi_{0}-\xi\right)\right\} \quad \forall(\xi, \eta) \in\left(\mathbf{R}_{s}^{9}\right)^{2}
$$

and $\Sigma(x, t):=\int_{0}^{t} \sigma(x, \tau) d \tau$, as $\alpha(\cdot, x)$ is maximal monotone we thus have

$$
\begin{aligned}
& \varphi(\sigma, \varepsilon-A(x): \Sigma, x)+\sigma: A(x): \Sigma \geq \sigma: \varepsilon \quad \forall \sigma, \varepsilon, \\
& \varphi(\sigma, \varepsilon-A(x): \Sigma, x)+\sigma: A(x): \Sigma=\sigma: \varepsilon \quad \Leftrightarrow \quad(1.1) .
\end{aligned}
$$

By integrating over $\Omega \times] 0, T$, this system yields

$$
\begin{aligned}
J(\sigma, \varepsilon):= & \iint_{\Omega \times] 0, T[}[\varphi(\sigma, \varepsilon-A(x): \Sigma, x)-\sigma: \varepsilon] d x d t \\
& +\frac{1}{2} \int_{\Omega} \Sigma: A(x):\left.\Sigma d x\right|_{t=T} \geq 0 \quad \forall \sigma, \varepsilon, \\
J(\sigma, \varepsilon)= & 0 \Leftrightarrow \quad(1.1) \text { in } \Omega \times] 0, T[.
\end{aligned}
$$

By (2.13), the latter statement also reads

$$
J(\sigma, \varepsilon)=\inf J \quad \Leftrightarrow \quad(1.1) \text { in } \Omega \times] 0, T[;
$$

actually, by (2.12), the infimum of $J$ necessarily vanishes, as $\alpha(\cdot, x)$ is maximal monotone. We may thus rewrite (1.1) in the form (1.7). 


\section{Weak formulation, existence and uniqueness}

In this section we provide the weak formulation of a boundary-value problem, that accounts for quasi-static processes in an inhomogeneous visco-elastic material represented by the constitutive relation (1.1). We then prove existence and uniqueness of the solution via classical techniques, see e.g. [38,50,71], in view of the ensuing modelling of composite materials.

P.D.E. and constitutive relation. Let us fix any $T>0$ and set $\left.A_{t}:=A \times\right] 0, t[$ for any subset $A$ of $\mathbf{R}^{3}$ and any $\left.\left.t \in\right] 0, T\right]$. Let us also assume that a load $\vec{f}_{1} \in L^{1}\left(\Omega_{T}\right)^{3}$ and a traction $\vec{g} \in L^{1}\left(\Gamma_{1 T}\right)$ are respectively applied to a domain $\Omega$ of $\mathbf{R}^{3}$ and to a part $\Gamma_{1}$ of its boundary, whereas the remainder $\Gamma_{0}$ of the boundary is kept fixed. (We select this homogeneous condition just for the sake of simplicity.) We shall neglect the inertia term, set the quasi-static force-balance equation

$$
-\nabla \cdot \sigma=\vec{f}_{1} \quad \text { in } \mathcal{D}^{\prime}\left(\Omega_{T}\right)^{3},
$$

and, denoting by $\vec{v}$ the outward-oriented unit normal vector on $\Gamma_{1}$, prescribe the boundary conditions

$$
\begin{array}{ll}
\vec{u}=\overrightarrow{0} & \text { a.e. on } \Gamma_{0 T}, \\
\sigma \cdot \vec{v}=\vec{g} & \text { a.e. on } \Gamma_{1 T} .
\end{array}
$$

We fix any $p, q$ such that $1<q \leq 2 \leq p<+\infty$ and $1 / p+1 / q=1$. We assume that

$$
\begin{aligned}
& \varphi: \mathbf{R}_{s}^{9} \times \mathbf{R}_{s}^{9} \times \Omega \rightarrow \mathbf{R} \cup\{+\infty\} \text { is a Borel function, and } \\
& \varphi(\cdot, \cdot, x) \text { represents a maximal monotone operator } \alpha(\cdot, x) \text {, for a.e. } x, \\
& \quad \exists c_{1}, \ldots, c_{4}>0: \forall v, z \in \mathbf{R}_{s}^{9}, \text { for a.e. } x \in \Omega, \\
& c_{1}\left(|v|^{p}+|z|^{q}\right)-c_{2} \leq \varphi(v, z, x) \leq c_{3}\left(|v|^{p}+|z|^{q}\right)+c_{4}, \\
& A \in L^{\infty}(\Omega)^{3^{4}}, \quad A \text { fulfills (2.3) and (2.4) a.e. in } \Omega .
\end{aligned}
$$

The reader will notice that we specified some regularity hypotheses in terms of the representative function, rather than the represented mapping $\alpha$.

Functional framework. Henceforth we assume that the domain $\Omega$ is bounded and of Lipschitz class, that $\Gamma_{0}$ is measurable and has positive bidimensional Hausdorff measure. Denoting the trace operator by $\gamma_{0}$, we also set

$$
V:=\left\{\vec{v} \in W^{1, q}(\Omega)^{3}: \gamma_{0} \vec{v}=\overrightarrow{0} \text { a.e. on } \Gamma_{0}\right\}, \quad\|\vec{v}\|_{V}:=\left\|\nabla^{s} \vec{v}\right\|_{L^{q}(\Omega)^{9}} .
$$

By the Korn and Poincaré inequalities (for the extension of the former to $W^{1, q}(\Omega)^{3}$ see e.g. $[46,84]), V$ is a closed Banach subspace of $W^{1, q}(\Omega)^{3}$. Identifying the dual space of $L^{q}(\Omega)^{3}$ with $L^{p}(\Omega)^{3}$ and denoting the dual space of $V$ by $V^{\prime}$, we get

$V \subset L^{q}(\Omega)^{3}, L^{p}(\Omega)^{3} \subset V^{\prime}$ with compact, continuous and dense injections. (3.8) 
We denote by $\langle\cdot, \cdot\rangle$ the duality pairing between $V^{\prime}$ and $V$, define the linear and continuous operator

$$
\nabla \cdot: L^{p}(\Omega)_{s}^{9} \rightarrow V^{\prime},\langle\nabla \cdot w, \vec{v}\rangle:=-\int_{\Omega} w: \nabla^{s} \vec{v} d x \quad \forall w \in L^{p}(\Omega)_{s}^{9}, \forall \vec{v} \in V
$$

and assume that

$$
\vec{f} \in L^{p}\left(0, T ; V^{\prime}\right) .
$$

Problem 3.1. (Weak formulation) Find $(\vec{u}, \sigma)$ such that, setting $\varepsilon:=\nabla^{s} \vec{u}$,

$$
\begin{gathered}
\vec{u} \in L^{q}(0, T ; V), \quad \sigma \in L^{p}\left(\Omega_{T}\right)_{s}^{9}, \\
\varepsilon-A(x): \int_{0}^{t} \sigma(x, \tau) d \tau \in \alpha(\sigma, x) \quad \text { a.e. in } \Omega_{T}, \\
\left.\int_{\Omega} \sigma: \nabla^{s} \vec{v} d x=\langle\vec{f}, \vec{v}\rangle \quad \forall \vec{v} \in V, \text { a.e. in }\right] 0, T[.
\end{gathered}
$$

For any $\vec{f}_{1} \in L^{p}\left(\Omega_{T}\right)^{3}$ and any $\vec{g} \in L^{p}\left(\Gamma_{1 T}\right)^{3}$, if we set

$$
\left.\langle\vec{f}, \vec{v}\rangle=\int_{\Omega} \vec{f}_{1} \cdot \vec{v} d x d \tau+\int_{\Gamma_{1}} \vec{g} \cdot \gamma_{0} \vec{v} d s \quad \forall \vec{v} \in V, \text { a.e. in }\right] 0, T[
$$

then the hypothesis (3.10) is fulfilled. By (3.13), $-\nabla \cdot \sigma=\vec{f}_{1}$ in $\mathcal{D}^{\prime}(\Omega)^{3}$, a.e. in ]0, $T$ [. Thus $\nabla \cdot \sigma \in L^{p}\left(\Omega_{T}\right)^{3}$ and this equation holds a.e. in $\Omega_{T}$.

Theorem 3.1 (Existence). Let $1<q \leq 2 \leq p<+\infty$ be such that $1 / p+1 / q=1$. If (3.4)-(3.6), (3.10) are fulfilled, then Problem 3.1 has a solution.

Proof.

(i) Approximation

Let us fix any $m \in \mathbf{N}$ and set

$$
h:=\frac{T}{m}, \quad \vec{f}_{m}^{n}:=\frac{1}{h} \int_{(n-1) h}^{n h} \vec{f}(t) d t \quad \text { in } V^{\prime}, \text { for } n=1, \ldots, m .
$$

Next we introduce a time-discretization of Problem 3.1.

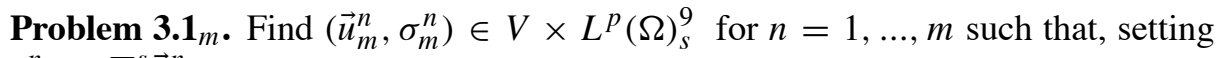
$\varepsilon_{m}^{n}:=\nabla^{s} \vec{u}_{m}^{n}$,

$$
\begin{array}{ll}
\varepsilon_{m}^{n}-h A(x): \sum_{i=1}^{n} \sigma_{m}^{i} \in \alpha\left(\sigma_{m}^{n}, x\right) & \text { a.e. in } \Omega, \\
\int_{\Omega} \sigma_{m}^{n}: \nabla^{s} \vec{v} d x=\left\langle\vec{f}_{m}^{n}, \vec{v}\right\rangle & \forall \vec{v} \in V .
\end{array}
$$


We shall prove existence of a solution of this problem step by step, via the classical theory of maximal monotone operators, $c f . e . g .[19,21,58,80,91]$. Let us set

$$
B_{m}^{n}(v, x):= \begin{cases}\alpha(v, x)+h A(x): v & \forall v \in \mathbf{R}_{s}^{9}, \text { for } n=1 \\ \alpha(v, x)+h A(x): v+h A(x): \sum_{i=1}^{n-1} \sigma_{m}^{i} & \forall v \in \mathbf{R}_{s}^{9}, \text { for } n=2, \ldots, m,\end{cases}
$$

so that (3.16) also reads $\sigma_{m}^{n}=\left(B_{m}^{n}\right)^{-1}\left(\varepsilon_{m}^{n}, x\right)$ a.e. in $\Omega$. The system (3.16), (3.17) is then equivalent to the quasilinear elliptic problem

$$
\vec{u}_{m}^{n} \in V, \quad-\nabla^{*} \cdot\left[\left(B_{m}^{n}\right)^{-1}\left(\nabla^{s} \vec{u}_{m}^{n}, x\right)\right]=\vec{f}_{m}^{n} \quad \text { in } V^{\prime} .
$$

The operator $B_{m}^{n}$ is maximal monotone, and the same applies to its inverse $\left(B_{m}^{n}\right)^{-1}$. By the hypotheses on $\varphi$ and $A$, the mapping $B_{m}^{n}$ is locally bounded. $\left(B_{m}^{n}\right)^{-1}$ is then coercive, in the sense that for any selection $w \in B_{m}^{n}(v, \cdot)$

$$
\frac{1}{\|v\|_{V}} \int_{\Omega} w: v d x \rightarrow+\infty \quad \text { as } \quad\|v\|_{V} \rightarrow+\infty .
$$

The equation (3.18) has then a solution $\vec{u}_{m}^{n}$, and this determines $\varepsilon_{m}^{n}=\nabla^{s} \vec{u}_{m}^{n}$ and $\sigma_{m}^{n}=\left(B_{m}^{n}\right)^{-1}\left(\varepsilon_{m}^{n}\right)$. Thus Problem $3.1_{m}$ has a solution.

For any family $\left\{v_{m}^{n}\right\}_{n=1, \ldots, m}$ of functions $\Omega \rightarrow \mathbf{R}$, let us set

$$
\begin{aligned}
& v_{m}:=\text { piecewise-linear time-interpolate of } v_{m}^{1}, \ldots, v_{m}^{m}, \text { a.e. in } \Omega, \\
& \left.\bar{v}_{m}(\cdot, t):=v_{m}^{n} \quad \text { a.e. in } \Omega, \forall t \in\right](n-1) h, n h[\text {, for } n=1, \ldots, m .
\end{aligned}
$$

Defining the piecewise-linear interpolate $\Sigma_{m}(x, t):=\int_{0}^{t} \bar{\sigma}_{m}(x, \tau) d \tau$ for a.e. $(x, t) \in$ $\Omega_{T}$ and the corresponding piecewise-constant interpolate $\bar{\Sigma}_{m}$, the system (3.16), (3.17) reads

$$
\begin{aligned}
\bar{\varepsilon}_{m}-A(x): \bar{\Sigma}_{m} \in \alpha\left(\bar{\sigma}_{m}, x\right) & \text { a.e. in } \Omega_{T}, \\
-\nabla^{*} \cdot \bar{\sigma}_{m}=\overline{\vec{f}}_{m} & \text { in } \left.V^{\prime}, \text { a.e. in }\right] 0, T[.
\end{aligned}
$$

(ii) A Priori estimates

By the Fitzpatrick property (2.12), the inclusion (3.20) is equivalent to

$$
\varphi\left(\bar{\sigma}_{m}, \bar{\varepsilon}_{m}-A(x): \bar{\Sigma}_{m}, x\right)=\bar{\sigma}_{m}: \bar{\varepsilon}_{m}-\bar{\sigma}_{m}: A(x): \bar{\Sigma}_{m} \quad \text { a.e. in } \Omega_{T} .
$$

Notice that (3.21) yields $\int_{\Omega} \bar{\sigma}_{m}: \bar{\varepsilon}_{m} d x=\left\langle\overline{\vec{f}}_{m}, \overline{\vec{u}}_{m}\right\rangle$ a.e. in ]0, $T$ [. By integrating (3.22) in space and time, we then get

$$
\begin{aligned}
& \frac{1}{2} \int_{\Omega} \bar{\Sigma}_{m}: A(x):\left.\bar{\Sigma}_{m} d x\right|_{\tau=t}+\iint_{\Omega_{t}} \varphi\left(\bar{\sigma}_{m}, \bar{\varepsilon}_{m}-A: \bar{\Sigma}_{m}, x\right) d x d \tau \\
& \left.\left.=\int_{0}^{t}\left\langle\overline{\vec{f}}_{m}, \overline{\vec{u}}_{m}\right\rangle d \tau \quad \forall t \in\right] 0, T\right] .
\end{aligned}
$$


Denoting the volume of $\Omega$ by $|\Omega|$, by (3.5) and (3.10) we then have

$$
\begin{aligned}
& c_{1} \iint_{\Omega_{t}}\left(\left|\bar{\sigma}_{m}\right|^{p}+\left|\bar{\varepsilon}_{m}-A(x): \bar{\Sigma}_{m}\right|^{q}\right) d x d \tau \\
& \left.\left.\leq\|\overrightarrow{\vec{f}}\|_{L^{p}\left(0, T ; V^{\prime}\right)}\left\|\overline{\vec{u}}_{m}\right\|_{L^{q}(0, t ; V)}+c_{2} t|\Omega| \quad \forall t \in\right] 0, T\right] .
\end{aligned}
$$

On the other hand, denoting by $C_{1}, C_{2}, \ldots$ suitable constants independent of $m$, by the Korn inequality and by (3.6), it is easy to see that

$$
\begin{aligned}
& \left\|\overline{\vec{u}}_{m}\right\|_{L^{q}(0, T ; V)} \leq C_{1}\left\|\bar{\varepsilon}_{m}\right\|_{L^{q}\left(\Omega_{T}\right)^{9}}, \\
& \iint_{\Omega_{t}}\left|A(x): \bar{\Sigma}_{m}\right|^{q} d x d \tau \leq C_{2} \iint_{\Omega_{t}}\left|\bar{\sigma}_{m}\right|^{q} d x d \tau \leq C_{3}\left(\iint_{\Omega_{t}}\left|\bar{\sigma}_{m}\right|^{p} d x d \tau\right)^{q / p} .
\end{aligned}
$$

We claim that then

$$
\left\|\overline{\vec{u}}_{m}\right\|_{L^{q}(0, T ; V)},\left\|\bar{\sigma}_{m}\right\|_{L^{p}\left(\Omega_{T}\right)^{9}} \leq C_{2}
$$

Actually, by (3.24) and (3.25),

$$
\begin{aligned}
& \left\|\overline{\vec{u}}_{m}\right\|_{L^{q}(0, t ; V)}^{q} \leq C_{1}^{q} \iint_{\Omega_{t}}\left|\bar{\varepsilon}_{m}\right|^{q} d x d \tau \\
\leq & 2^{q} C_{1}^{q} \max \left\{1, C_{3}\right\}\left\{\left(\iint_{\Omega_{t}}\left|\bar{\sigma}_{m}\right|^{p} d x d \tau\right)^{q / p}+\iint_{\Omega_{t}}\left(\left|\bar{\varepsilon}_{m}-A(x): \bar{\Sigma}_{m}\right|^{q}\right) d x d \tau\right\} \\
\leq & 2^{q} C_{1}^{q} \max \left\{1, C_{3}\right\}\left\{1+\iint_{\Omega_{t}}\left(\left|\bar{\sigma}_{m}\right|^{p}+\left|\bar{\varepsilon}_{m}-A(x): \bar{\Sigma}_{m}\right|^{q}\right) d x d \tau\right\} \\
\leq & \left.\left.2^{q} C_{1}^{q} \max \left\{1, C_{3}\right\}\left\{1+c_{1}^{-1}\left(\|\overline{\vec{f}}\|_{L^{p}\left(0, T ; V^{\prime}\right)}\left\|\overline{\vec{u}}_{m}\right\|_{L^{q}(0, t ; V)}+c_{2} t|\Omega|\right)\right\} \forall t \in\right] 0, T\right] .
\end{aligned}
$$

The uniform estimate for $u_{m}$ follows, and by (3.24) we then get the estimate for $\sigma_{m}$.

(iii) Passage to the limit

By the uniform estimates (3.26) there exist $\vec{u}$ and $\sigma$ such that, setting $\varepsilon:=\nabla^{s} \vec{u}$, as $m \rightarrow \infty$ along a suitable sequence ${ }^{(1)}$

$$
\begin{array}{ll}
\overline{\vec{u}}_{m} \rightarrow \vec{u} & \text { in } L^{q}(0, T ; V), \\
\bar{\sigma}_{m} \rightarrow \sigma & \text { in } L^{p}\left(\Omega_{T}\right)^{9},
\end{array}
$$

whence, setting $\Sigma(x, t):=\int_{0}^{t} \sigma(x, \tau) d \tau$ for a.e. $(x, t) \in \Omega_{T}$,

$$
\begin{array}{lr}
A(x): \bar{\Sigma}_{m} \rightarrow A(x): \Sigma & \text { in } L^{p}\left(\Omega_{T}\right)^{9}, \\
\bar{\varepsilon}_{m} \rightarrow \varepsilon & \text { in } L^{q}\left(\Omega_{T}\right)^{9} .
\end{array}
$$

${ }^{(1)} \mathrm{By} \rightarrow$ and $\rightarrow$ we shall denote strong and weak convergence, respectively. 
Notice that the left side of (3.23) is a convex and lower semicontinuous functional of $\bar{\sigma}_{m}$ and $\bar{\varepsilon}_{m}$. By passing to the limit in (3.21) and to the inferior limit in (3.23), we then get (3.13) and

$$
\begin{aligned}
\frac{1}{2} \int_{\Omega} \sigma: A(x):\left.\sigma d x\right|_{\tau=t} & +\iint_{\Omega_{t}} \varphi(\sigma, \varepsilon-A: \Sigma, x) d x d \tau \\
& \left.\left.\leq \int_{0}^{t}\langle\vec{f}, \vec{u}\rangle d \tau \quad \forall t \in\right] 0, T\right],
\end{aligned}
$$

namely,

$$
\left.\left.\iint_{\Omega_{t}}[\sigma: A(x): \Sigma+\varphi(\sigma, \varepsilon-A(x): \Sigma, x)] d x d \tau \leq \int_{0}^{t}\langle\vec{f}, \vec{u}\rangle d \tau \quad \forall t \in\right] 0, T\right] .
$$

By the next Proposition we get (3.12), so that we can conclude that $(\sigma, \vec{u})$ solves Problem 3.1.

Let us denote by $I_{B}$ the indicator function of any set $B$, i.e.,

$$
I_{B}(v):=0 \quad \text { if } v \in B, \quad I_{B}(v):=+\infty \quad \text { otherwise. }
$$

Proposition 3.2. Under the hypotheses of Theorem 3.1, let us set

$$
\begin{aligned}
\mathcal{X}_{\vec{f}}:= & \left\{\sigma \in L^{p}\left(\Omega_{T}\right)_{s}^{9}:-\nabla \cdot \sigma=\vec{f} \quad \text { in } V^{\prime} \text {, a.e. in }\right] 0, T[\} \\
J_{0}(\vec{u}, \sigma):= & \iint_{\Omega_{T}}[\sigma: A(x): \Sigma+\varphi(\sigma, \varepsilon-A(x): \Sigma, x)] d x d t \\
& -\int_{0}^{T}\langle\vec{f}, \vec{u}\rangle d t+I_{\mathcal{X}_{\vec{f}}}(\sigma) \quad \forall(\vec{u}, \sigma) \in L^{q}(0, T ; V) \times L^{p}\left(\Omega_{T}\right)_{s}^{9} .
\end{aligned}
$$

For any $(\vec{u}, \sigma) \in L^{q}(0, T ; V) \times L^{p}\left(\Omega_{T}\right)_{s}^{9}$, then

$$
(\vec{u}, \sigma) \text { solves Problem } 3.1 \quad \Leftrightarrow \quad J_{0}(\vec{u}, \sigma)=\inf J_{0}=0 .
$$

Proof. For any $(\vec{u}, \sigma) \in L^{q}(0, T ; V) \times \mathcal{X}_{\vec{f}},\langle\vec{f}, \vec{u}\rangle=\int_{\Omega} \sigma: \varepsilon d x$ a.e. in $] 0, T[$. Hence

$$
\begin{aligned}
J_{0}(\vec{u}, \sigma)= & \iint_{\Omega_{T}}[\sigma:(A(x): \Sigma-\varepsilon)+\varphi(\sigma, \varepsilon-A(x): \Sigma, x)] d x d t+I_{\mathcal{X}_{\vec{f}}}(\sigma) \\
& \forall(\vec{u}, \sigma) \in L^{q}(0, T ; V) \times L^{p}\left(\Omega_{T}\right)_{s}^{9} .
\end{aligned}
$$

It then suffices to notice that, by (2.11) and (2.12), the integrand is a.e. nonnegative, and vanishes if and only if (3.12) is fulfilled. 
Theorem 3.3 (Uniqueness). Let the hypotheses of Theorem 3.1 be fulfilled and $(\vec{u}, \sigma)$ be a solution of Problem 3.1. Assume that

either $\alpha(\cdot, x)$ is strictly increasing for a.e. $x \in \Omega$,

or $A(x)$ is positive-definite for a.e. $x \in \Omega$.

Then $\sigma$ is uniquely determined. If $\alpha(\cdot, x)$ is single-valued for a.e. $x \in \Omega$, then $\vec{u}$ is also unique.

Proof. For $i=1,2$ let $\left(\vec{u}_{i}, \sigma_{i}\right)$ be a solution of Problem 3.1, set $\varepsilon_{i}:=\nabla^{s} \vec{u}_{i}$, $\overline{\vec{u}}:=\vec{u}_{1}-\vec{u}_{2}$, and define $\bar{\varepsilon}, \bar{\sigma}$ similarly a.e. in $\Omega_{T}$. Let us now write (3.12) for $i=1,2$ multiply the difference of these inclusions by $\bar{\sigma}$, and integrate in space and time. Selecting a suitable $\bar{r} \in \alpha\left(\sigma_{1}, \cdot\right)-\alpha\left(\sigma_{2}, \cdot\right)$ a.e. in $\Omega_{T}$ and setting $\bar{\Sigma}(\cdot, t):=$ $\int_{0}^{t} \bar{\sigma}(\cdot, \tau) d \tau$ for a.e. $\left.t \in\right] 0, T[$, this yields

$$
\begin{array}{r}
\iint_{\Omega_{t}} \bar{\sigma}: \bar{\varepsilon} d x d \tau \geq \frac{1}{2} \int_{\Omega} \bar{\Sigma}(x, t): A(x): \bar{\Sigma}(x, t) d x+\iint_{\Omega_{t}} \bar{r}: \bar{\sigma} d x d \tau \\
\text { a.e. in }] 0, T[.
\end{array}
$$

On the other hand by selecting $\vec{v}=\overline{\vec{u}}$ in (3.13) we have $\iint_{\Omega_{t}} \bar{\sigma}: \bar{\varepsilon} d x d \tau=0$. By (3.39), we then infer that $\bar{\sigma}=0$ a.e. in $\Omega_{T}$; thus $\sigma$ is uniquely determined. If $\alpha(\cdot, x)$ is single-valued for a.e. $x \in \Omega$, then $\varepsilon$ is also unique by (3.12). By the Korn inequality the same then applies to $\vec{u}$.

Remark. Under the hypotheses of Theorem 3.1 the inclusion (3.12) might be reformulated as $\varepsilon \in \mathcal{A}(\sigma)$, for an operator $\mathcal{A}$ that is maximal monotone in $L^{2}\left(\Omega_{T}\right)_{S}^{9}$. One might then use the corresponding Fitzpatrick function. This reformulation would not lead to a substantial modification of the above results.

\section{Two-scale limit}

In this section we deal with the asymptotic behaviour of a periodic composite material. After briefly recalling the notion of two-scale convergence, we derive a twolength-scale model by letting the functions $\alpha$ and $A$ oscillate more and more rapidly in space.

Two-scale weak formulation. By a classical procedure, first we introduce a reference volume element $Y:=\left[0,1{ }^{3}\right.$, and denote by $\mathcal{Y}$ the same set equipped with the topological and differential structure of the unit torus. Any $Y$-periodic function defined on $\mathbf{R}^{3}$ may thus be identified with a function defined on $\mathcal{Y}$. We then fix a positive parameter $\eta<<1$, assume that the constitutive functions $\varphi$ and $A$ depend $(\eta Y)$-periodically on $x$; the same then applies to the maximal monotone operator $\alpha$ that is represented by $\varphi$. We accordingly replace the constitutive relation (1.1) by

$$
\varepsilon-A(x / \eta): \Sigma \in \alpha(\sigma, x / \eta) \quad \text { a.e. in } \Omega_{T}\left(\Sigma(x, t):=\int_{0}^{t} \sigma(x, \tau) d \tau\right) .
$$


In order to enforce the $Y$-periodicity, we may equivalently set $y=x / \eta$ modulus $Y$, and let $y$ range through the unit torus $\mathcal{Y}$. We are thus induced to consider the two-scale relation

$$
\varepsilon-A(y): \Sigma \in \alpha(\sigma, y) \quad \text { a.e. in } \Omega_{T} \times \mathcal{Y} .
$$

Like in (2.12), this inclusion is tantamount to the inequality

$$
\varphi(\sigma, \varepsilon-A(y): \Sigma, y) \leq(\varepsilon-A(y): \Sigma): \sigma \quad \text { a.e. in } \Omega_{T} \times \mathcal{Y} .
$$

As $A$ and $\alpha$ explicitly depend on $y$ but not on $x$, this inclusion may be interpreted as the constitutive behaviour of a macroscopically homogeneous but mesoscopically nonhomogeneous material. The next developments might trivially be extended to a material that is also macroscopically inhomogeneous, just by allowing $A$ and $\alpha$ to depend explicitly on the pair $(x, y)$.

For any integrable function $v=v(y)$ we define the average component $\hat{v}$ and the fluctuating component $\widetilde{v}$ :

$$
\hat{v}:=\int_{\mathcal{Y}} v(y) d y, \quad \tilde{v}:=v-\hat{v} \quad \forall v \in L^{1}(\mathcal{Y}) .
$$

We shall assume that the hypotheses (3.4)-(3.6) hold with $y \in \mathcal{Y}$ in place of $x \in \Omega$, and that, instead of (3.10),

$$
\vec{f} \in L^{p}\left(\Omega_{T}\right)^{3} .
$$

We couple the inclusion (4.1) with the force-balance equation (3.13), that here reads

$$
\left.\int_{\Omega} \sigma: \nabla^{s} \vec{v} d x=\int_{\Omega} \vec{f} \cdot \vec{v} d x \quad \forall \vec{v} \in V, \text { a.e. in }\right] 0, T[,
$$

and formulate a single-length-scale problem, that we label by Problem $3.1_{\eta}$. This only differs from Problem 3.1 in two respects: here the argument $x$ is replaced by $x / \eta$ in the functions $A$ and $\alpha$, and the homogeneous Neumann condition is implicitly assumed on $\Gamma_{1 T}$, in order to simplify the homogenization procedure. By Theorem 3.1 for any $\eta>0$ this problem has a solution $\left(\vec{u}_{\eta}, \sigma_{\eta}\right)$, and by (3.26)

$$
\left.\left\|\vec{u}_{\eta}\right\|_{L^{q}(0, T ; V)},\left\|\sigma_{\eta}\right\|_{L^{p}\left(\Omega_{T}\right)^{9}} \leq \text { Constant (independent of } \eta\right) .
$$

Next we formulate a two-scale problem, that we shall then retrieve by passing to the limit in Problem $3.1_{\eta}$.

Problem 4.1 (Two-scale formulation). Find

$$
\vec{u} \in L^{q}(0, T ; V), \quad \vec{u}_{1} \in L^{q}\left(\Omega_{T} ; W^{1, q}(\mathcal{Y})^{3}\right), \quad \sigma \in L^{p}\left(\Omega_{T} \times \mathcal{Y}\right)_{s}^{9},
$$

such that $\hat{\vec{u}}_{1}=\overrightarrow{0}$ a.e. in $\Omega_{T}$, and, setting $\varepsilon=\nabla^{s} \vec{u}+\nabla_{y}^{s} \vec{u}_{1}$ a.e. in $\Omega_{T} \times \mathcal{Y}$,

$$
\begin{array}{ll}
\varepsilon-A(y): \Sigma \in \alpha(\sigma, y) & \text { a.e. in } \Omega_{T} \times \mathcal{Y}, \\
\int_{\Omega} \hat{\sigma}: \nabla^{s} \vec{v} d x=\int_{\Omega} \vec{f} \cdot \vec{v} d x & \forall \vec{v} \in V, \text { a.e. in }] 0, T[, \\
\nabla_{y} \cdot \sigma=\overrightarrow{0} & \text { in } \mathcal{D}^{\prime}(\mathcal{Y})^{3} \text {, a.e. in } \Omega_{T} .
\end{array}
$$


Two-scale convergence. In view of relating Problem $3.1_{\eta}$ to Problem 4.1, we briefly review the definition of two-scale convergence along the lines of $[4,73]$. For any $r \in] 1,+\infty$ [ we say that a bounded sequence $\left\{u_{\eta}\right\}$ of $L^{r}(\Omega)$ (weakly) two-scale converges to $u \in L^{r}(\Omega \times \mathcal{Y})$ in the latter space, and write $u_{\eta} \underset{2}{\rightarrow} u$, whenever

$$
\int_{\Omega} u_{\eta}(x) v(x, x / \eta) d x \rightarrow \iint_{\Omega \times \mathcal{Y}} u(x, y) v(x, y) d x d y \quad \forall v \in \mathcal{D}(\Omega \times \mathcal{Y}) .
$$

We extend this definition to space- and time-dependent functions as follows. For any $r, s \in] 1,+\infty$ [, any bounded sequence $\left\{u_{\eta}\right\}$ of $L^{s}\left(0, T ; L^{r}(\Omega)\right)$ and any $u \in$ $L^{s}\left(0, T ; L^{r}(\Omega \times \mathcal{Y})\right)$, we say that $u_{\eta} \underset{2}{\longrightarrow} u$ whenever

$$
\begin{array}{r}
\iint_{\Omega_{T}} u_{\eta}(x, t) v(x, x / \eta, t) d x d t \rightarrow \iiint_{\Omega_{T} \times \mathcal{Y}} u(x, y, t) v(x, y, t) d x d y d t \\
\forall v \in \mathcal{D}\left(\Omega_{T} \times \mathcal{Y}\right) .
\end{array}
$$

The extension to either vector- or tensor-valued functions is obvious. In view of the next statements, we remind the reader that we assumed $\Omega$ to be a (bounded) Lipschitz domain.

Lemma $4.1([4,73])$. Let $r \in] 1,+\infty\left[\right.$ and $\left\{u_{\eta}\right\}$ be a bounded sequence of $L^{r}(\Omega)$. Then there exists $u \in L^{r}(\Omega \times \mathcal{Y})$ such that, possibly extracting a subsequence,

$$
u_{\eta} \underset{2}{\vec{n}} u \quad \text { in } L^{r}(\Omega \times \mathcal{Y}) \text {. }
$$

Lemma 4.2 ([86]). Let $r \in] 1,+\infty\left[, \vec{u} \in L^{r}(\Omega)^{9}\right.$, and a sequence $\left\{\vec{u}_{\eta}\right\}$ of $W^{1, r}(\Omega)^{3}$ be such that $\vec{u}_{\eta} \rightarrow \vec{u}$ in this space. Then there exists $\vec{u}_{1} \in L^{r}\left(\Omega ; W^{1, r}(\mathcal{Y})^{3}\right)$ such that $\hat{\vec{u}}_{1}=\overrightarrow{0}$ a.e. in $\Omega$, and, possibly extracting a subsequence,

$$
\nabla^{s} \vec{u}_{\eta} \underset{2}{\rightarrow} \nabla^{s} \vec{u}+\nabla_{y}^{s} \vec{u}_{1} \quad \text { in } L^{r}(\Omega \times \mathcal{Y})^{9}
$$

Lemma $4.3([4,73])$. Let $r \in] 1,+\infty\left[\right.$ and a bounded sequence $\left\{w_{\eta}\right\}$ of $L^{r}(\Omega)_{s}^{9}$ be such that $\left\{\eta \nabla \cdot w_{\eta}\right\}$ is bounded in $L^{r}(\Omega)^{3}$. Then there exists $w \in L^{r}(\Omega \times \mathcal{Y})_{s}^{9}$ such that $\nabla_{y} \cdot w \in L^{r}(\Omega \times \mathcal{Y})^{3}$, and, possibly extracting a subsequence,

$$
w_{\eta} \underset{2}{\rightarrow} w \quad \text { in } L^{r}(\Omega \times \mathcal{Y})_{s}^{9}, \quad \eta \nabla \cdot w_{\eta} \underset{2}{\rightarrow} \nabla_{y} \cdot w \quad \text { in } L^{r}(\Omega \times \mathcal{Y})^{3} .
$$

Derivation of Problem 4.1. Next we go back to Problem 3.1 $\eta$ and prove two-scale convergence to a solution of Problem 4.1.

Theorem 4.4 (Existence and uniqueness). Let $1<q \leq 2 \leq p<+\infty$ be such that $1 / p+1 / q=1$, and let the hypotheses (3.4)-(3.6) and (4.4) be fulfilled, here with $y \in \mathcal{Y}$ in place of $x \in \Omega$. For any $\eta>0$ let $\left(\vec{u}_{\eta}, \sigma_{\eta}\right)$ be a solution of 
Problem 3.1 $1_{\eta}$, and assume the uniform estimates (4.5) (in Section 3 we saw that such a family of solutions exists). Then there exist $\vec{u}, \vec{u}_{1}, \sigma$ as in (4.6) such that, setting $\varepsilon_{\eta}=\nabla^{s} \vec{u}_{\eta}$ and $\varepsilon=\nabla^{s} \vec{u}+\nabla_{y}^{s} \vec{u}_{1}$, as $\eta$ vanishes along a suitable sequence,

$$
\begin{array}{lc}
\vec{u}_{\eta} \rightarrow \vec{u} & \text { in } L^{q}(0, T ; V), \\
\sigma_{\eta}{ }_{2} \sigma & \text { in } L^{p}\left(\Omega_{T} \times \mathcal{Y}\right)^{9}, \\
\varepsilon_{\eta}{ }_{2} \varepsilon & \text { in } L^{q}\left(\Omega_{T} \times \mathcal{Y}\right)^{9} .
\end{array}
$$

This entails that $\left(\vec{u}, \vec{u}_{1}, \sigma\right)$ is a solution of Problem 4.1 .

If moreover (3.39) (here with $y$ in place of $x$ ) is fulfilled a.e. in $\mathcal{Y}$, then $\sigma$ is uniquely determined. If $\alpha(\cdot, y)$ is single-valued for a.e. $y \in \mathcal{Y}$, then $\vec{u}$ is also unique.

Proof. (i) First we prove convergence and existence of a solution. By (4.5) and by Lemmata 4.1 and 4.2, there exist $\vec{u}, \vec{u}_{1}, \sigma, \varepsilon$ such that (4.15)-(4.17) are fulfilled. By taking $\eta \rightarrow 0$ in $(3.13)_{\eta}$ (namely, (3.13) written for $\sigma_{\eta}$-we shall repeatedly use this notation) we obviously retrieve (4.8). By Lemma 4.3 the equation (4.9) also follows from $(3.13)_{\eta}$. In view of deriving (4.7), let us notice that $(3.33)_{\eta}$ yields

$$
\begin{aligned}
\iint_{\Omega_{T}} & {\left[\sigma_{\eta}: A(x / \eta): \Sigma_{\eta}+\varphi\left(\sigma_{\eta}, \varepsilon_{\eta}-A(x / \eta): \Sigma_{\eta}, x / \eta\right)\right] d x d t } \\
& \leq \iint_{\Omega_{T}} \vec{f} \cdot \vec{u}_{\eta} d x d t
\end{aligned}
$$

As the semicontinuity properties of weak (single-scale) convergence take over to weak two-scale convergence, see Section 1 of [88], we have

$$
\begin{aligned}
& \liminf _{\eta \rightarrow 0} \iint_{\Omega_{T}} \sigma_{\eta}: A(x / \eta): \Sigma_{\eta} d x d t \geq \frac{1}{2} \iint_{\Omega^{* \mathcal{Y}}} \Sigma: A(y):\left.\Sigma d x d y\right|_{t=T} \\
&=\iiint_{\Omega_{T} \times \mathcal{Y}} \sigma: A(y): \Sigma d x d y d t \\
& \liminf _{\eta \rightarrow 0} \iint_{\Omega_{T}} \varphi\left(\sigma_{\eta}, \varepsilon_{\eta}-A(x / \eta): \Sigma_{\eta}, x / \eta\right) d x d t \\
& \geq \iiint_{\Omega_{T} \times \mathcal{Y}} \varphi(\sigma, \varepsilon-A(y): \Sigma, y) d x d y d t .
\end{aligned}
$$

By passing to the inferior limit in (4.18) we then get

$$
\iiint_{\Omega_{T} \times \mathcal{Y}}[\sigma: A(y): \Sigma+\varphi(\sigma, \varepsilon-A(y): \Sigma, y)] d x d y d t \leq \iint_{\Omega_{T}} \vec{f} \cdot \vec{u} d x d t .
$$


On the other hand by (4.8) and (4.9)

$$
\begin{aligned}
& \iint_{\Omega_{T}} \hat{\sigma}: \hat{\varepsilon} d x d t=\iint_{\Omega_{T}} \vec{f} \cdot \vec{u} d x d t \\
& \begin{aligned}
\int_{\mathcal{Y}} \sigma: \varepsilon d y & =\int_{\mathcal{Y}} \sigma: \nabla^{s} \vec{u} d y+\int_{\mathcal{Y}} \sigma: \nabla_{y}^{s} \vec{u}_{1} d y \\
& =\hat{\sigma}: \int_{\mathcal{Y}} \nabla^{s} \vec{u} d y=\hat{\sigma}: \hat{\varepsilon} \quad \text { a.e. in } \Omega_{T},
\end{aligned}
\end{aligned}
$$

so that (4.19) also reads

$$
\iiint_{\Omega_{T} \times \mathcal{Y}}\{\sigma:[A(y): \Sigma-\varepsilon]+\varphi(\sigma, \varepsilon-A(y): \Sigma, y)\} d x d y d t \leq 0 .
$$

As by (2.13) the opposite inequality holds for any $(\sigma, \varepsilon)$, this inequality is actually an equality, and by (2.14) it is equivalent to (4.7). Thus Problem 4.1 has a solution.

The statement about uniqueness follows by the argument of Theorem 3.3.

\section{Single-scale homogenization of the constitutive law}

In view of homogenizing the complete Problem 4.1 in the next section, here we deal with a cell problem, and integrate the constitutive relation (4.7) over the reference volume element $\mathcal{Y}$.

In the first part of this section we replace $A(y): \Sigma(x, y, t)$ by $S(x, y, t)$, and regard the functions $\sigma$ and $S$ as mutually independent. After this replacement, in (4.7) the coarse-scale variable $x$ and the time $t$ just play the role of parameters; we then omit them, and deal with the stationary inclusion

$$
\varepsilon(y)-S(y) \in \alpha(\sigma(y), y) \quad \text { for a.e. } y \in \mathcal{Y} \text {. }
$$

Afterwards we shall reintroduce the space- and time-dependence, and replace $S$ by $A(y): \Sigma$.

Throughout this section we still assume the hypotheses (3.4)-(3.6) with $y \in \mathcal{Y}$ in place of $x \in \Omega$, and with $1<q \leq 2 \leq p<+\infty, 1 / p+1 / q=1$. We shall also use the decomposition (4.3). Next we state a result that we shall apply afterwards.

Lemma 5.1. Let $B_{1}, B_{2}$ be real Banach spaces and $B_{2}$ be also reflexive. Let a function $\left.f: B_{1} \times B_{2} \rightarrow\right]-\infty,+\infty$ ] be prescribed, and define the function

$$
g: B_{1} \rightarrow[-\infty,+\infty]: \xi_{1} \mapsto \inf \left\{f\left(\xi_{1}, \xi_{2}\right): \xi_{2} \in B_{2}\right\}
$$

Then:

(i) if $f$ is convex then $g$ is also convex; 
(ii) if $f$ is sequentially weakly lower semicontinuous and "locally-in- $B_{1}$ and uniformly-in- $B_{2}$ coercive", in the sense that

$$
\begin{aligned}
& \forall \text { bounded set } S \subset B_{1}, \forall M \in \mathbf{R}, \\
& \text { the set }\left\{\xi_{2} \in B_{2}: f\left(\xi_{1}, \xi_{2}\right) \leq M, \forall \xi_{1} \in S\right\} \text { is bounded, }
\end{aligned}
$$

then $g$ is also sequentially weakly lower semicontinuous.

(iii) If $f$ is coercive, that is, $\left\{\left(\xi_{1}, \xi_{2}\right) \in B_{1} \times B_{2}: f\left(\xi_{1}, \xi_{2}\right) \leq M\right\}$ is bounded for any $M>0$, then $g$ is also coercive.

Proof. (We reproduce the argument of [[87]] for the sake of completeness.)

(i) For any $\xi_{1}^{\prime}, \xi_{1}^{\prime \prime} \in B_{1}$, any $\xi_{2}^{\prime}, \xi_{2}^{\prime \prime} \in B_{2}$ and any $\left.\lambda \in\right] 0,1[$, the convexity of $f$ yields

$$
\begin{aligned}
g\left(\lambda \xi_{1}^{\prime}+(1-\lambda) \xi_{1}^{\prime \prime}\right) & \leq f\left(\lambda \xi_{1}^{\prime}+(1-\lambda) \xi_{1}^{\prime \prime}, \lambda \xi_{2}^{\prime}+(1-\lambda) \xi_{2}^{\prime \prime}\right) \\
& =f\left(\lambda\left(\xi_{1}^{\prime}, \xi_{2}^{\prime}\right)+(1-\lambda)\left(\xi_{1}^{\prime \prime}, \xi_{2}^{\prime \prime}\right)\right) \\
& \leq \lambda f\left(\xi_{1}^{\prime}, \xi_{2}^{\prime}\right)+(1-\lambda) f\left(\xi_{1}^{\prime \prime}, \xi_{2}^{\prime \prime}\right)
\end{aligned}
$$

By taking the infimum with respect to $\xi_{2}^{\prime}$ and $\xi_{2}^{\prime \prime}$, we then get

$$
g\left(\lambda \xi_{1}^{\prime}+(1-\lambda) \xi_{1}^{\prime \prime}\right) \leq \lambda g\left(\xi_{1}^{\prime}\right)+(1-\lambda) g\left(\xi_{1}^{\prime \prime}\right) .
$$

Thus $g$ is convex.

(ii) Let us fix any sequence $\left\{\xi_{1 n}\right\}$ in $B_{1}$ that weakly converges to some $\xi_{1} \in B_{1}$; thus $\left\{\xi_{1 n}\right\}$ is bounded. If $L:=\lim _{n \rightarrow \infty} \inf _{n \rightarrow \infty} g\left(\xi_{1 n}\right)=+\infty$ then trivially $g\left(\xi_{1}\right) \leq L$. Let us then assume that $L<+\infty$. By definition of $g$ there exists a sequence $\left\{\xi_{2 n}\right\}$ in $B_{2}$ such that

$$
\begin{array}{lll}
f\left(\xi_{1 n}, \xi_{2 n}\right) \leq g\left(\xi_{1 n}\right)+1 / n & \forall n \in \mathbf{N} \quad \text { if } L>-\infty \\
f\left(\xi_{1 n}, \xi_{2 n}\right) \leq-n & \forall n \in \mathbf{N} \quad \text { if } L=-\infty .
\end{array}
$$

By (5.2) the sequence $\left\{\xi_{2 n}\right\}$ is bounded in $B_{2}$, hence there exists $\xi_{2} \in B_{2}$ such that $\xi_{2 n} \rightarrow \xi_{2}$ weakly in $B_{2}$, as $n \rightarrow \infty$ along a further subsequence. By passing to the limit in (5.3) along this subsequence, the sequential weak lower semicontinuity of $f$ then yields

$$
g\left(\xi_{1}\right) \leq f\left(\xi_{1}, \xi_{2}\right) \leq \liminf _{n \rightarrow \infty} f\left(\xi_{1 n}, \xi_{2 n}\right) \leq \liminf _{n \rightarrow \infty} g\left(\xi_{1 n}\right) .
$$

(iii) The final statement about coerciveness is straightforward. 
Next we shall derive a relation for the average fields $\hat{\varepsilon}, \hat{\sigma}, \hat{S}$ from the inclusion (5.1); this integrated formulation will then turn out to be equivalent to the original one, in a sense that we shall specify. We proceed through a number of steps.

(i) Let us first set

$$
\begin{aligned}
& W:=\left\{\eta \in L^{p}(\mathcal{Y})_{s}^{9}: \hat{\eta}=0, \nabla \cdot \eta=\overrightarrow{0} \text { in } \mathcal{D}^{\prime}(\mathcal{Y})^{3}\right\}, \\
& Z:=\left\{\zeta \in L^{q}(\mathcal{Y})_{s}^{9}: \hat{\zeta}=0, \zeta=\nabla^{s} \vec{v} \text { a.e. in } \mathcal{Y} \text {, for some } \vec{v} \in W^{1, q}(\mathcal{Y})^{3}\right\},
\end{aligned}
$$

and note the orthogonality properties

$$
\begin{array}{ll}
\int_{\mathcal{Y}} \eta(y): \zeta(y) d y=0 & \forall \eta \in Z, \forall \zeta \in W, \\
\int_{\mathcal{Y}} \hat{\eta}: \tilde{\zeta}(y) d y=\int_{\mathcal{Y}} \tilde{\eta}: \hat{\zeta}(y) d y=0 & \forall \eta \in L^{p}(\mathcal{Y})^{9}, \forall \zeta \in L^{q}(\mathcal{Y})^{9},
\end{array}
$$

whence

$$
\int_{\mathcal{Y}} \eta(y): \zeta(y) d y=\hat{\eta}: \hat{\zeta} \quad \forall \eta \in \mathbf{R}_{s}^{9}+Z, \forall \zeta \in \mathbf{R}_{s}^{9}+W .
$$

(ii) Defining $\varphi$ as in (2.10), with $y \in \mathcal{Y}$ in place of $x \in \Omega$, (2.11) and (2.12) respectively yield

$$
\varphi(\sigma, \varepsilon-S, y) \geq \sigma:(\varepsilon-S) \quad \forall(\sigma, \varepsilon, S) \in\left(\mathbf{R}_{s}^{9}\right)^{3}, \text { a.e. in } \mathcal{Y},
$$

and, for any $(\sigma, \varepsilon, S) \in\left(\mathbf{R}_{s}^{9}+W\right) \times\left(\mathbf{R}_{s}^{9}+Z\right) \times\left(\mathbf{R}_{s}^{9}+W\right)$,

$$
\text { (5.1) } \Leftrightarrow \varphi(\sigma, \varepsilon-S, y)=\sigma:(\varepsilon-S) \quad \text { a.e. in } \mathcal{Y} .
$$

(iii) For any $(\sigma, \varepsilon, S) \in\left(\mathbf{R}_{s}^{9}+W\right) \times\left(\mathbf{R}_{s}^{9}+Z\right) \times\left(\mathbf{R}_{s}^{9}+W\right)$, (5.7) yields

$$
\varphi(\sigma, \varepsilon-S, y)+\sigma: S \geq(\hat{\sigma}+\widetilde{\sigma}):(\hat{\varepsilon}+\widetilde{\varepsilon}) \quad \text { a.e. in } \mathcal{Y} .
$$

By (3.5) the integral

$$
\Psi(\sigma, \varepsilon, S):=\int_{\mathcal{Y}}[\varphi(\sigma, \varepsilon-S, y)+\sigma: S] d y
$$

is finite. By (5.6) and (5.9), we have

$$
\Psi(\sigma, \varepsilon, S) \geq \hat{\sigma}: \hat{\varepsilon} \quad \forall(\sigma, \varepsilon, S) \in\left(\mathbf{R}_{s}^{9}+W\right) \times\left(\mathbf{R}_{s}^{9}+Z\right) \times\left(\mathbf{R}_{s}^{9}+W\right) .
$$

(iv) We claim that

$$
\text { (5.1) } \Leftrightarrow \quad \Psi(\sigma, \varepsilon, S)=\hat{\sigma}: \hat{\varepsilon} .
$$


The implication " $\Rightarrow$ " follows from (5.8) by integration because of (5.6). Next we derive the opposite implication. As $\int_{\mathcal{Y}} \sigma: \varepsilon d y=\hat{\sigma}: \hat{\varepsilon}$ by (5.6), the equality $\Psi(\sigma, \varepsilon, S)=\hat{\sigma}: \hat{\varepsilon}$ also reads

$$
\int_{\mathcal{Y}}[\varphi(\sigma, \varepsilon-S, y)+\sigma: S-\sigma: \varepsilon] d y=0 .
$$

By (5.7) this integrand is pointwise nonnegative, therefore it vanishes a.e. in $\mathcal{Y}$; by (2.14) this is tantamount to (5.1).

(v) Next we introduce the dependence on time and replace $S$ by $A(y): \Sigma$ a.e. in $\mathcal{Y}_{T}$, namely, we deal with the inclusion

$$
\begin{aligned}
& \varepsilon(y, t)-A(y): \Sigma(y, t) \in \alpha(\sigma(y, t), y) \\
& \text { for a.e. }(y, t) \in \mathcal{Y}_{T}, \text { with } \Sigma(y, t):=\int_{0}^{t} \sigma(y, \tau) d \tau .
\end{aligned}
$$

Let us define the functional

$$
\begin{aligned}
& \Lambda_{[0, T]}: L^{p}(0, T)_{s}^{9} \times L^{q}(0, T)_{s}^{9} \rightarrow \mathbf{R}:(\bar{\sigma}, \bar{\varepsilon}) \mapsto \\
& \inf \left\{\int_{0}^{T} \Psi\left(\bar{\sigma}+\widetilde{\sigma}, \bar{\varepsilon}+\widetilde{\varepsilon}, A(y): \int_{0}^{t}[\bar{\sigma}(\tau)+\widetilde{\sigma}(y, \tau)] d \tau\right) d t:\right. \\
& \left.\tilde{\sigma} \in L^{p}(0, T ; W), \widetilde{\varepsilon} \in L^{q}(0, T ; Z)\right\} .
\end{aligned}
$$

(Most often we shall write $\Lambda$ in place of $\Lambda_{[0, T]}$.) This infimum is finite, by our hypotheses on $\varphi$ and $A$. The inequality (5.11) and the statement (5.12) respectively yield

$$
\begin{gathered}
\Lambda(\bar{\sigma}, \bar{\varepsilon}) \geq \int_{0}^{T} \bar{\sigma}: \bar{\varepsilon} d t \quad \forall \bar{\sigma} \in L^{p}(0, T)_{s}^{9}, \forall \bar{\varepsilon} \in L^{q}(0, T)_{s}^{9}, \\
(5.13) \Rightarrow \quad \Lambda(\hat{\sigma}, \hat{\varepsilon}) \int_{0}^{T} \hat{\sigma}: \hat{\varepsilon} d t .
\end{gathered}
$$

(vi) Next we invert the latter implication. Let $\bar{\sigma} \in L^{p}(0, T)_{S}^{9}$ and $\bar{\varepsilon} \in L^{q}(0, T)_{S}^{9}$ be such that

$$
\Lambda(\bar{\sigma}, \bar{\varepsilon})=\int_{0}^{T} \bar{\sigma}: \bar{\varepsilon} d t \quad \text { (or equivalently, } \Lambda(\bar{\sigma}, \bar{\varepsilon}) \leq \int_{0}^{T} \bar{\sigma}: \bar{\varepsilon} d t \text {, by (5.15)). }
$$

Let us define the Banach spaces $B_{1}:=L^{p}(0, T){ }_{s}^{9} \times L^{q}(0, T)_{s}^{9}, B_{2}:=L^{p}(0, T ; W) \times$ $L^{q}(0, T ; Z)$, and the functional

$$
\begin{aligned}
& F: B_{1} \times B_{2} \rightarrow \mathbf{R}:(\bar{\sigma}, \bar{\varepsilon}, \widetilde{\sigma}, \widetilde{\varepsilon}) \\
& \mapsto \int_{0}^{T} \Psi\left(\bar{\sigma}+\widetilde{\sigma}, \bar{\varepsilon}+\widetilde{\varepsilon}, A(y): \int_{0}^{t}[\bar{\sigma}(\tau)+\widetilde{\sigma}(y, \tau)] d \tau\right) d t,
\end{aligned}
$$


so that $(5.14)$ reads

$$
\begin{gathered}
\Lambda(\bar{\sigma}, \bar{\varepsilon})=\inf \left\{F(\bar{\sigma}, \bar{\varepsilon}, \widetilde{\sigma}, \widetilde{\varepsilon}): \widetilde{\sigma} \in L^{p}(0, T ; W), \widetilde{\varepsilon} \in L^{q}(0, T ; Z)\right\} \\
\forall(\bar{\sigma}, \bar{\varepsilon}) \in L^{p}(0, T)_{s}^{9} \times L^{q}(0, T)_{s}^{9} .
\end{gathered}
$$

Note that $F$ is convex and lower semicontinuous, and by (3.5) it is locally uniformly coercive in the sense of (5.2). Therefore the infimum of (5.14) is attained; namely, there exist $\widetilde{\sigma} \in L^{p}(0, T ; W)$ and $\widetilde{\varepsilon} \in L^{q}(0, T ; Z)$ such that, setting $\sigma=\bar{\sigma}+\tilde{\sigma}$, $\varepsilon=\bar{\varepsilon}+\widetilde{\varepsilon}$ and $\Sigma=\int_{0}^{t}[\bar{\sigma}(\tau)+\widetilde{\sigma}(y, \tau)] d \tau$,

$$
\Lambda(\bar{\sigma}, \bar{\varepsilon})=F(\bar{\sigma}, \bar{\varepsilon}, \widetilde{\sigma}, \widetilde{\varepsilon})=\int_{0}^{T} \Psi(\sigma, \varepsilon, A(y): \Sigma) d t=\int_{0}^{T} \bar{\sigma}: \bar{\varepsilon} d t
$$

By (5.11) we then deduce that $\Psi(\sigma, \varepsilon, A(y): \Sigma)=\bar{\sigma}: \bar{\varepsilon}$ a.e. in $] 0, T$, and (5.12) yields (5.13).

Finally, by Lemma 5.1 the functional $\Lambda$ is also convex, lower semicontinuous and coercive.

We have thus proved the next statement.

Theorem 5.2. Let $1<q \leq 2 \leq p<+\infty$ be such that $1 / p+1 / q=1$, assume that the hypotheses (3.4)-(3.6) are fulfilled with $y \in \mathcal{Y}$ in place of $x \in \Omega$, and define $\Lambda$ as in (5.14). Then:

(i) The functional $\Lambda$ is convex, lower semicontinuous and coercive.

$$
\Lambda(\bar{\sigma}, \bar{\varepsilon}) \geq \int_{0}^{T} \bar{\sigma}: \bar{\varepsilon} d t \quad \forall \bar{\sigma} \in L^{p}\left(\mathcal{Y}_{T}\right)_{s}^{9}, \forall \bar{\varepsilon} \in L^{q}(0, T)_{s}^{9} .
$$

(iii) For any $\sigma \in L^{p}\left(0, T ; W+\mathbf{R}_{s}^{9}\right)$ and any $\varepsilon \in L^{q}\left(0, T ; Z+\mathbf{R}_{s}^{9}\right)$, (using the notation (4.3))

$$
\varepsilon-A(y): \int_{0}^{t} \sigma(y, \tau) d \tau \in \alpha(\sigma, y) \text { a.e. in } \mathcal{Y}_{T} \Rightarrow \Lambda(\hat{\sigma}, \hat{\varepsilon}) \leq \int_{0}^{T} \hat{\sigma}: \hat{\varepsilon} d t
$$

The latter is actually an equality because of (5.20); it is thus equivalent to a nullminimization problem:

$\Lambda(\hat{\sigma}, \hat{\varepsilon}) \leq \int_{0}^{T} \hat{\sigma}: \hat{\varepsilon} d t \Leftrightarrow J_{0}(\hat{\sigma}, \hat{\varepsilon})=\inf _{L^{p}(0, T)_{s}^{9} \times L^{q}(0, T)_{s}^{9}} J_{0}=0$,

where $J_{0}(\bar{\sigma}, \bar{\varepsilon}):=\Lambda(\bar{\sigma}, \bar{\varepsilon})-\int_{0}^{T} \bar{\sigma}: \bar{\varepsilon} d t \quad \forall(\bar{\sigma}, \bar{\varepsilon}) \in L^{p}(0, T)_{s}^{9} \times L^{q}(0, T)_{s}^{9}$. 
(iv) Conversely, for any pair $(\bar{\sigma}, \bar{\varepsilon}) \in L^{p}(0, T)_{s}^{9} \times L^{q}(0, T)_{s}^{9}$,

$$
\begin{aligned}
& \Lambda(\bar{\sigma}, \bar{\varepsilon}) \leq \int_{0}^{T} \bar{\sigma}: \bar{\varepsilon} d t \Rightarrow \\
& \left\{\begin{array}{l}
\exists(\widetilde{\sigma}, \widetilde{\varepsilon}) \in L^{p}(0, T ; W) \times L^{q}(0, T ; Z) \text { such that } \\
\bar{\varepsilon}+\widetilde{\varepsilon}-A(y): \int_{0}^{t}[\bar{\sigma}(\tau)+\widetilde{\sigma}(y, \tau)] d \tau \in \alpha(\bar{\sigma}+\widetilde{\sigma}, y) \text { a.e. in } \mathcal{Y}_{T} .
\end{array}\right.
\end{aligned}
$$

(v) If (3.39) (here with $y$ in place of $x$ ) is fulfilled a.e. in $\mathcal{Y}$, then in part (iv) $\widetilde{\sigma}$ is uniquely determined. If $\alpha(\cdot, y)$ is single-valued for a.e. $y$, then $\widetilde{\varepsilon}$ is also unique.

The final statement can be checked by mimicking the uniqueness argument of Theorem 4.4. The only difference is that here there is no dependence on the coarse-scale variable $x \in \Omega$.

Ahead in Theorem 5.4 we shall see that $\Lambda(\bar{\sigma}, \bar{\varepsilon}) \leq \int_{0}^{T} \bar{\sigma}: \bar{\varepsilon} d t$ defines a maximal monotone relation between $\bar{\sigma}$ and $\bar{\varepsilon}$. By Fitzpatrick's Theorem 2.1 this entails that inf $J_{0}=0$, so that this inequality is equivalent to the minimization of $J_{0}$ (that is, the minimum necessarily vanishes).

Next we characterize the solution of the minimization problem (5.14). For the sake of readability, in the next statement we shall denote by $\partial_{i} \varphi$ the subdifferential of $\varphi$ with respect to the $i$ th argument $(i=1,2)$, and set $\Sigma(y, t):=\int_{0}^{t} \sigma(y, \tau) d \tau$.

Proposition 5.3. For any $(\sigma, \varepsilon) \in L^{p}\left(\mathcal{Y}_{T}\right)_{S}^{9} \times L^{q}\left(\mathcal{Y}_{T}\right)_{s}^{9}$, using the notation (4.3), (5.10) and (5.14),

$$
\Lambda(\hat{\sigma}, \hat{\varepsilon})=\int_{0}^{T} \Psi\left(\sigma, \varepsilon, A(y): \int_{0}^{t} \sigma(y, \tau) d \tau\right) d t<+\infty
$$

if and only if $(\sigma, \varepsilon) \in L^{p}\left(0, T ; \mathbf{R}_{s}^{9}+W\right) \times L^{q}\left(0, T ; \mathbf{R}_{s}^{9}+Z\right)$, and (dropping the argument $(\sigma, \varepsilon-A(y): \Sigma, y))$

$$
\begin{cases}\iint_{\mathcal{Y}_{T}}\left[\partial_{1} \varphi:(\sigma-w)-\partial_{2} \varphi: A(y): \int_{0}^{t}(\sigma-w)(y, \tau) d \tau\right. \\ \quad+(\sigma-w): A(y): \int_{0}^{t} \sigma(y, \tau) d \tau & \\ \left.\quad+\sigma: A(y): \int_{0}^{t}(\sigma-w)(y, \tau) d \tau\right] d y d t \leq 0 & \forall w \in L^{p}(0, T ; W), \\ \iint_{\mathcal{Y}_{T}} \partial_{2} \varphi:(\varepsilon-z) d y d t \leq 0 & \forall z \in L^{q}(0, T ; Z) .\end{cases}
$$

Remark. By part (iv) of Theorem 5.2, for any pair $(\bar{\sigma}, \bar{\varepsilon}) \in L^{p}(0, T)_{s}^{9} \times L^{q}(0, T)_{s}^{9}$, setting $\varepsilon=\bar{\varepsilon}+\widetilde{\varepsilon}$ and $\sigma=\bar{\sigma}+\widetilde{\sigma}$, the equation (5.25) determines the unknown $(\widetilde{\sigma}, \widetilde{\varepsilon}) \in L^{p}(0, T ; W) \times L^{q}(0, T ; Z)$. This defines a (possibly multivalued) operator

$$
\Xi: L^{p}(0, T)_{s}^{9} \times L^{q}(0, T)_{s}^{9} \rightarrow \mathcal{P}\left(L^{p}(0, T ; W) \times L^{q}(0, T ; Z)\right):(\bar{\sigma}, \bar{\varepsilon}) \mapsto(\widetilde{\sigma}, \widetilde{\varepsilon}) .
$$


In the terminology of [40],

$$
Q: L^{p}\left(0, T ; W+\mathbf{R}_{s}^{9}\right) \times L^{q}\left(0, T ; Z+\mathbf{R}_{s}^{9}\right) \rightarrow L^{p}(0, T)_{s}^{9} \times L^{q}(0, T)_{s}^{9}:
$$

$(\sigma, \varepsilon) \mapsto(\hat{\sigma}, \hat{\varepsilon})$ is a compression operator,

$$
R: L^{p}(0, T)_{s}^{9} \times L^{q}(0, T)_{s}^{9} \rightarrow \mathcal{P}\left(L^{p}\left(0, T ; W+\mathbf{R}_{s}^{9}\right) \times L^{q}\left(0, T ; Z+\mathbf{R}_{s}^{9}\right)\right):
$$

$$
(\bar{\sigma}, \bar{\varepsilon}) \mapsto(\sigma, \varepsilon)=(\bar{\sigma}, \bar{\varepsilon})+\Xi(\bar{\sigma}, \bar{\varepsilon}) \text { is a reconstruction operator, }
$$

(Clearly, here it is the information provided by the fine-scale fields $\widetilde{\sigma}$ and $\widetilde{\varepsilon}$ that are either compressed or reconstructed.) Indeed $Q \circ R=I$ and $R \circ Q \supset I$ by Theorem 5.2. Moreover $R=Q^{-1}$ under the hypotheses of part (iv) of that theorem.

Next we express the coarse-scale constitutive behaviour via a maximal monotone operator that acts in the space $L^{p}(0, T)_{s}^{9}$, and may thus exhibit dependence on the past (memory effect),

Theorem 5.4. Let $1<q \leq 2 \leq p<+\infty$ be such that $1 / p+1 / q=1$, assume that the hypotheses (3.4)-(3.6) are fulfilled with $y \in \mathcal{Y}$ in place of $x \in \Omega$, define the functional $\Lambda$ as in (5.14), and set

$$
\beta_{[0, T]}(\bar{\sigma}):=\left\{\bar{\varepsilon} \in L^{q}(0, T)_{s}^{9}: \Lambda(\bar{\sigma}, \bar{\varepsilon})=\int_{0}^{T} \bar{\sigma}: \bar{\varepsilon} d t\right\} \quad \forall \bar{\sigma} \in L^{p}(0, T)_{s}^{9} .
$$

This defines a maximal monotone operator $L^{p}(0, T)_{S}^{9} \rightarrow \mathcal{P}\left(L^{q}(0, T)_{S}^{9}\right)$.

The functional $\Lambda$ thus represents the operator $\beta_{[0, T]}$, that we shall also denote by $\beta$.

Proof. (i) First we show that $\beta$ is monotone, that is, for any $\left(\bar{\sigma}_{i}, \bar{\varepsilon}_{i}\right) \in L^{p}(0, T)_{s}^{9} \times$ $L^{q}(0, T)_{s}^{9}(i=1,2)$,

$$
\Lambda\left(\bar{\sigma}_{i}, \bar{\varepsilon}_{i}\right) \leq \int_{0}^{T} \bar{\sigma}_{i}: \bar{\varepsilon}_{i} d t(i=1,2) \quad \Rightarrow \quad \int_{0}^{T}\left(\bar{\sigma}_{1}-\bar{\sigma}_{2}\right):\left(\bar{\varepsilon}_{1}-\bar{\varepsilon}_{2}\right) d t \geq 0
$$

Here we use the argument of [42]. By the convexity of $\Lambda$ and by (5.28)

$$
\begin{aligned}
\Lambda\left(\frac{1}{2}\left(\bar{\sigma}_{1}+\bar{\sigma}_{2}\right), \frac{1}{2}\left(\bar{\varepsilon}_{1}+\bar{\varepsilon}_{2}\right)\right) & \leq \frac{1}{2} \Lambda\left(\bar{\sigma}_{1}, \bar{\varepsilon}_{1}\right)+\frac{1}{2} \Lambda\left(\bar{\sigma}_{2}, \bar{\varepsilon}_{2}\right) \\
& \leq \frac{1}{2} \int_{0}^{T} \bar{\sigma}_{1}: \bar{\varepsilon}_{1} d t+\frac{1}{2} \int_{0}^{T} \bar{\sigma}_{2}: \bar{\varepsilon}_{2} d t
\end{aligned}
$$

On the other hand (5.20) yields

$$
\frac{1}{4} \int_{0}^{T}\left(\bar{\sigma}_{1}+\bar{\sigma}_{2}\right):\left(\bar{\varepsilon}_{1}+\bar{\varepsilon}_{2}\right) d t \leq \Lambda\left(\frac{1}{2}\left(\bar{\sigma}_{1}+\bar{\sigma}_{2}\right), \frac{1}{2}\left(\bar{\varepsilon}_{1}+\bar{\varepsilon}_{2}\right)\right)
$$


By the two latter formulas we get

$$
\frac{1}{4} \int_{0}^{T}\left(\bar{\sigma}_{1}+\bar{\sigma}_{2}\right):\left(\bar{\varepsilon}_{1}+\bar{\varepsilon}_{2}\right) d t \leq \frac{1}{2} \int_{0}^{T}\left(\bar{\sigma}_{1}: \bar{\varepsilon}_{1}+\bar{\sigma}_{2}: \bar{\varepsilon}_{2}\right) d t,
$$

that is, $\int_{0}^{T}\left(\bar{\sigma}_{1}-\bar{\sigma}_{2}\right):\left(\bar{\varepsilon}_{1}-\bar{\varepsilon}_{2}\right) d t \geq 0$.

(ii) The maximality follows from [90, Theorem 5.6].

Lemma 5.5. If the mapping $\varphi(v, z, y)$ is strictly convex with respect to $v(z$, resp.) for any $z(v$, resp.), uniformly for $y \in \mathcal{Y}$, then the functional $\Lambda(\cdot, \bar{\varepsilon})(\Lambda(\bar{\sigma}, \cdot)$, resp.) is strictly convex for any admissible $\bar{\varepsilon}(\bar{\sigma}$, resp.). If the mapping $\varphi(\cdot, \cdot, y)$ is strictly convex uniformly for $y \in \mathcal{Y}$, then the functional $\Lambda$ is globally strictly convex.

Proof. Let us assume that $\varphi(\cdot, \cdot, y)$ is strictly convex uniformly for $y \in \mathcal{Y}$, and prove that $\Lambda$ is globally strictly convex. This hypothesis entails that $F$ (defined in (5.17)) is globally strictly convex. For any pair $\left(\bar{\sigma}_{i}, \bar{\varepsilon}_{i}\right) \in \operatorname{Dom}(\Lambda)(i=1,2)$ there exist $\left(\widetilde{\sigma}_{i}, \widetilde{\varepsilon}_{i}\right)$ such that, setting $\sigma_{i}:=\bar{\sigma}_{i}+\widetilde{\sigma}_{i}$ and $\varepsilon_{i}:=\bar{\varepsilon}_{i}+\widetilde{\varepsilon}_{i}$, we have

$$
\left(\bar{\sigma}_{i}, \widetilde{\sigma}_{i}, \bar{\varepsilon}_{i}, \widetilde{\varepsilon}_{i}\right) \in \operatorname{Dom}(F), \quad \Lambda\left(\bar{\sigma}_{i}, \bar{\varepsilon}_{i}\right)=F\left(\bar{\sigma}_{i}, \widetilde{\sigma}_{i}, \bar{\varepsilon}_{i}, \widetilde{\varepsilon}_{i}\right) \quad(i=1,2) .
$$

Let us now fix any $\lambda \in] 0,1\left[\right.$, set $\bar{\sigma}_{\lambda}:=\lambda \bar{\sigma}_{1}+(1-\lambda) \bar{\sigma}_{2}$, and define $\widetilde{\sigma}_{\lambda}, \bar{\varepsilon}_{\lambda}, \widetilde{\varepsilon}_{\lambda}$ similarly. If $\left(\bar{\sigma}_{1}, \bar{\varepsilon}_{1}\right) \neq\left(\bar{\sigma}_{2}, \bar{\varepsilon}_{2}\right)$, by $(5.18)$ and by the strict convexity of $F$ we deduce that

$$
\begin{aligned}
\Lambda\left(\bar{\sigma}_{\lambda}, \bar{\varepsilon}_{\lambda}\right) \leq F\left(\bar{\sigma}_{\lambda}, \widetilde{\sigma}_{\lambda}, \bar{\varepsilon}_{\lambda}, \widetilde{\varepsilon}_{\lambda}\right) & <\lambda F\left(\bar{\sigma}_{1}, \widetilde{\sigma}_{1}, \bar{\varepsilon}_{1}, \widetilde{\varepsilon}_{1}\right)+(1-\lambda) F\left(\bar{\sigma}_{2}, \widetilde{\sigma}_{2}, \bar{\varepsilon}_{2}, \widetilde{\varepsilon}_{2}\right) \\
& =\lambda \Lambda\left(\bar{\sigma}_{1}, \bar{\varepsilon}_{1}\right)+(1-\lambda) \Lambda\left(\bar{\sigma}_{2}, \bar{\varepsilon}_{2}\right) .
\end{aligned}
$$

The proof of the first statement is similar and is omitted.

Proposition 5.6. Let $1<q \leq 2 \leq p<+\infty$ be such that $1 / p+1 / q=1$, assume that the hypotheses (3.4)-(3.6) are fulfilled with $y \in \mathcal{Y}$ in place of $x \in \Omega$, and define $\varphi, \Lambda$ and $\beta$ as in (2.10), (5.14) and (5.28). If the mapping $\varphi(v, z, y)$ is strictly convex with respect to $z$ ( $v$, respectively) for any $v$ ( $z$, respectively), uniformly for $y \in \mathcal{Y}$, then the operator $\beta\left(\beta^{-1}\right.$, respectively) is single-valued.

Proof. In view of proving that $\beta$ is single-valued, let us assume that $\varphi(v, \cdot, y)$ is strictly convex for any $v$, uniformly for $y \in \mathcal{Y}$. Let us fix any $\bar{\sigma} \in \operatorname{Dom}(\beta)$, any $\lambda \in] 0,1\left[\right.$, any $\bar{\varepsilon}_{1}, \bar{\varepsilon}_{2} \in \beta(\bar{\sigma})$, and set $\bar{\varepsilon}_{\lambda}:=\lambda \bar{\varepsilon}_{1}+(1-\lambda) \bar{\varepsilon}_{2}$. As by Lemma 5.5 $\Lambda(\bar{\sigma}, \cdot)$ is strictly convex, if $\bar{\varepsilon}_{1} \neq \bar{\varepsilon}_{2}$ then

$$
\begin{aligned}
\Lambda\left(\bar{\sigma}, \bar{\varepsilon}_{\lambda}\right) & <\lambda \Lambda\left(\bar{\sigma}, \bar{\varepsilon}_{1}\right)+(1-\lambda) \Lambda\left(\bar{\sigma}, \bar{\varepsilon}_{2}\right) \\
& \stackrel{(5.28)}{=} \lambda \int_{0}^{T} \bar{\sigma}: \bar{\varepsilon}_{1} d t+(1-\lambda) \int_{0}^{T} \bar{\sigma}: \bar{\varepsilon}_{2} d t=\int_{0}^{T} \bar{\sigma}: \bar{\varepsilon}_{\lambda} d t .
\end{aligned}
$$

Thus $\Lambda\left(\bar{\sigma}, \bar{\varepsilon}_{\lambda}\right)<\int_{0}^{T} \bar{\sigma}: \bar{\varepsilon}_{\lambda} d t$, at variance with (5.20). We then conclude that the operator $\beta$ is single-valued.

The proof of the single-valuedness of $\beta^{-1}$ is similar and is omitted. 
Causality. The upscaled relation $\beta$ acts between spaces of time-dependent functions. It may thus account for nonlocal dependence in time: whenever $\bar{\varepsilon} \in \beta(\bar{\sigma})$, for any $\tau \in[0, T]$, a priori the tensor $\bar{\varepsilon}(\tau)$ might depend on the tensor-function $\bar{\sigma}$ in the whole interval $[0, T]$. This raises the issue of causality. However, because (5.20), it is easily seen that (using the notation (5.14))

$$
\Lambda_{[0, T]}(\bar{\sigma}, \bar{\varepsilon})=\int_{0}^{T} \bar{\sigma}: \bar{\varepsilon} d t \Rightarrow \Lambda_{[0, \tau]}(\bar{\sigma}, \bar{\varepsilon})=\int_{0}^{\tau} \bar{\sigma}: \bar{\varepsilon} d t \quad \forall \tau \in[0, T] .
$$

We have thus proved the next statement.

Proposition 5.7 (Causality). Let $\beta$ be defined as in (5.28). Then for any $\bar{\sigma} \in$ $L^{p}(0, T)_{s}^{9}$ and any $\bar{\varepsilon} \in L^{q}(0, T)_{s}^{9}$,

$$
\left.\bar{\varepsilon} \in \beta_{[0, T]}(\bar{\sigma}) \quad \Rightarrow \quad \bar{\varepsilon}\right|_{[0, \tau]} \in \beta_{[0, \tau]}\left(\left.\bar{\sigma}\right|_{[0, \tau]}\right) \quad \forall \tau \in[0, T],
$$

namely, $\bar{\varepsilon}_{[0, \tau]}$ only depends on $\bar{\sigma}_{[0, \tau]}$.

\section{Single-scale homogenization of the whole problem}

In this section we derive a single-scale problem, and show its equivalence to the two-scale Problem 4.1, in a sense that we shall specify. This entails that if a sequence of solutions of Problem $3.1_{\eta}$ two-scale converges to a solution of Problem 4.1, then it also single-scale converges to a solution of the homogenized coarsescale problem of this section. We also show that, conversely, any solution of the latter problem can be retrieved in this way. We then retrieve these results via $\Gamma$ convergence. Finally, we briefly discuss the significance of analogical models.

First we introduce the single-scale problem, and then relate it to the two-scale formulation. We still assume that $1<q \leq 2 \leq p<+\infty, 1 / p+1 / q=1$, that the hypotheses (3.4)-(3.6) and (4.4) are fulfilled with $y \in \mathcal{Y}$ in place of $x \in \Omega$, and that the spaces $W, Z$ and the functional $\Lambda$ are as in (5.4) and (5.14). We still denote the average over $\mathcal{Y}$ by the $h a t$, and use the bar to label several functions that might occur as averages of functions that depend on $y$ : for instance, $\bar{\sigma}$ is a candidate to be represented in the form $\bar{\sigma}=\int_{\mathcal{Y}} \sigma(y) d y(=: \hat{\sigma})$. On the other hand this does not apply to $\vec{u}$, which cannot be the average of any fine-scale field.

Problem 6.1. Find $\vec{u} \in L^{q}(0, T ; V)$ and $\bar{\sigma} \in L^{p}\left(\Omega_{T}\right)_{s}^{9}$ such that, setting $\bar{\varepsilon}:=$ $\nabla^{s} \vec{u}$

$$
\begin{array}{ll}
\bar{\varepsilon} \in \beta(\bar{\sigma}) & \text { in } L^{2}\left(\Omega_{T}\right)_{s}^{9}, \\
\int_{\Omega} \bar{\sigma}: \nabla \vec{v} d x=\int_{\Omega} \vec{f} \cdot \vec{v} d x & \forall \vec{v} \in V, \text { a.e. in }] 0, T[.
\end{array}
$$


By Theorem 5.4 the operator $\beta$ is maximal monotone, the inclusion (6.1) is equivalent to

$$
\Lambda(\bar{\sigma}, \bar{\varepsilon}) \leq \int_{0}^{T} \bar{\sigma}: \bar{\varepsilon} d t \quad \text { a.e. in } \Omega .
$$

Moreover, by Theorem 5.2 this holds if and only if there exist $\widetilde{\sigma} \in L^{p}\left(\Omega_{T} ; W\right)$ and $\widetilde{\varepsilon} \in L^{q}\left(\Omega_{T} ; Z\right)$ such that

$$
\bar{\varepsilon}+\widetilde{\varepsilon}-A(y): \int_{0}^{t}[\bar{\sigma}(\tau)+\widetilde{\sigma}(\cdot, \cdot, \tau)] d \tau \in \alpha(\bar{\sigma}+\widetilde{\sigma}, y) \quad \text { a.e. in } \Omega_{T} \times \mathcal{Y} \text {. }
$$

Problem 6.1 may thus be regarded as a homogenized version of Problem 4.1.

The next statement may be compared with Proposition 3.2.

Proposition 6.1. Let $1<q \leq 2 \leq p<+\infty, 1 / p+1 / q=1$, assume that the hypotheses (3.4)-(3.6) and (4.4) are fulfilled, here with $y \in \mathcal{Y}$ in place of $x \in \Omega$. Let us define $\mathcal{X}_{\vec{f}}$ and its indicator function $I_{\mathcal{X}_{\vec{f}}}$ as in (3.34) and (3.35), and set

$$
\begin{array}{r}
J_{1}(\vec{u}, \bar{\sigma}):=\int_{\Omega} \Lambda\left(\bar{\sigma}, \nabla^{s} \vec{u}\right) d x-\iint_{\Omega_{T}} \vec{f} \cdot \vec{u} d x d t+I_{\mathcal{X}_{\vec{f}}}(\bar{\sigma}) \\
\forall(\vec{u}, \bar{\sigma}) \in L^{q}(0, T ; V) \times L^{p}\left(\Omega_{T}\right)_{s}^{9} .
\end{array}
$$

Then

$$
(\vec{u}, \bar{\sigma}) \text { solves Problem } 6.1 \quad \Leftrightarrow \quad J_{1}(\vec{u}, \bar{\sigma})=\inf J_{1}=0 .
$$

Proof. It suffices to notice that

$$
\iint_{\Omega_{T}} \bar{\sigma}: \nabla^{s} \vec{u} d x d t=\iint_{\Omega_{T}} \vec{f} \cdot \vec{u} d x d t \quad \forall(\vec{u}, \bar{\sigma}) \in L^{q}(0, T ; V) \times \mathcal{X}_{\vec{f}},
$$

that by part (ii) of Theorem 5.2

$$
\Lambda(\bar{\sigma}, \bar{\varepsilon}) \geq \int_{0}^{T} \bar{\sigma}: \bar{\varepsilon} d t \quad \text { a.e. in } \Omega, \forall \bar{\sigma} \in L^{p}\left(\Omega_{T}\right)_{s}^{9}, \forall \bar{\varepsilon} \in L^{q}\left(\Omega_{T}\right)_{s}^{9},
$$

and that the opposite inequality is tantamount to the inclusion (6.1).

Next we assume that $\alpha(\cdot, y)$ is single-valued, that the hypotheses of Theorem 5.2 are fulfilled, and establish a precise one-to-one relation between the solutions of Problems 4.1 and 6.1, using the decomposition (4.3). By part (iv) of Theorem 5.2, for any $(\vec{u}, \bar{\sigma}) \in L^{q}(0, T ; V) \times L^{p}\left(\Omega_{T}\right)_{s}^{9}$ that satisfies (6.1) there exists $(\widetilde{\sigma}, \widetilde{\varepsilon}) \in L^{p}\left(\Omega_{T} ; W\right) \times L^{q}\left(\Omega_{T} ; Z\right)$ such that $\varepsilon:=\nabla^{s} \vec{u}+\widetilde{\varepsilon}$ and $\sigma:=\bar{\sigma}+\widetilde{\sigma}$ fulfill the inclusion $\varepsilon-A: \sigma \in \alpha(\sigma, y)$ a.e. in $\Omega_{T} \times \mathcal{Y}$. Moreover, by Lemma 4.2 there exists $\vec{u}_{1} \in L^{q}\left(\Omega_{T} ; W^{1, q}(\mathcal{Y})^{3}\right)$ such that $\hat{\vec{u}}_{1}=\overrightarrow{0}$ a.e. in $\Omega_{T}$ and $\widetilde{\varepsilon}=\nabla_{y}^{s} \vec{u}_{1}$. This defines a multivalued mapping

$$
\begin{aligned}
& R_{0}: L^{q}(0, T ; V) \times L^{p}\left(\Omega_{T}\right)_{s}^{9} \\
& \rightarrow \mathcal{P}\left(L^{q}(0, T ; V) \times L^{q}\left(\Omega_{T} ; W^{1, q}(\mathcal{Y})^{3}\right) \times L^{p}\left(\Omega_{T} \times \mathcal{Y}\right)_{S}^{9}\right): \\
& \quad(\vec{u}, \bar{\sigma}) \mapsto\left(\vec{u}, \vec{u}_{1}, \sigma\right) .
\end{aligned}
$$


Theorem 6.2. Let $1<q \leq 2 \leq p<+\infty$ be such that $1 / p+1 / q=1$, and let the hypotheses (3.4) - (3.6) and (4.4) be fulfilled, here with $y \in \mathcal{Y}$ in place of $x \in \Omega$. Let also $\alpha(\cdot, y)$ be single-valued for a.e. $y \in \mathcal{Y}$. Then:

(i) If $\left(\vec{u}, \vec{u}_{1}, \sigma\right)$ is a solution of Problem 4.1 (by Theorem 4.4 such a solution exists), then $(\vec{u}, \hat{\sigma})$ solves Problem 6.1 .

(ii) Conversely, if $(\vec{u}, \bar{\sigma})$ is a solution of Problem 6.1 , then any $\left(\vec{u}, \vec{u}_{1}, \sigma\right) \in R_{0}(\vec{u}, \bar{\sigma})$ solves Problem 4.1.

(iii) If (3.39) (here with $y$ in place of $x$ ) is fulfilled, then the solution of Problem 6.1 is unique.

Proof. (i) Let us remind the definitions (3.36) and (6.4) of the functionals $J_{0}$ and $J_{1}$. If $\left(\vec{u}, \vec{u}_{1}, \sigma\right)$ is a solution of Problem 4.1 , then $J_{0}(\vec{u}, \hat{\sigma})=0$ by Propositions 3.2. Hence $J_{1}(\vec{u}, \hat{\sigma})=0$ by the definition (5.14) of $\Lambda$. By Propositions $6.1,(\vec{u}, \hat{\sigma})$ thus solves Problem 6.1.

(ii) This part follows from the definition of the operator $R_{0}$.

(iii) By Theorem 4.4, Problem 4.1 has only one solution. The equivalence between Problems 4.1 and 6.1 then yields the uniqueness of the solution of the latter problem.

Corollary 6.3. Let $1<q \leq 2 \leq p<+\infty$ be such that $1 / p+1 / q=1$, and let the hypotheses (3.4)-(3.6) and (4.4) be fulfilled, here with $y \in \mathcal{Y}$ in place of $x \in \Omega$. Then there exist $\vec{u}$ and $\bar{\sigma} \in L^{p}\left(\Omega_{T}\right)_{s}^{9}$ such that, as $\eta \rightarrow 0$ along a suitable sequence,

$$
\begin{array}{ll}
\vec{u}_{\eta} \rightarrow \vec{u} & \text { in } L^{q}(0, T ; V), \\
\sigma_{\eta} \rightarrow \bar{\sigma} & \text { in } L^{p}\left(\Omega_{T}\right)^{9} .
\end{array}
$$

This entails that $(\vec{u}, \bar{\sigma})$ is a solution of Problem 6.1. If (3.39) (here with y in place of $x)$ is fulfilled and $\alpha(\cdot, y)$ is single-valued for a.e. $y \in \mathcal{Y}$, then the whole sequences converge.

Proof. Theorem 4.4 yields (4.15)-(4.17) as $\eta \rightarrow 0$ along a suitable sequence. Notice that (6.7) coincides with (4.15), and (4.16) yields (6.8) for $\bar{\sigma}=\hat{\sigma}$. By part (i) of Theorem 6.2 the pair $(\vec{u}, \bar{\sigma})$ solves Problem 6.1. The final statement is a direct consequence of the uniqueness of the solution, that is stated in part (iii) of Theorem 6.2.

Remark. By Theorem 6.2 the average operator $Q_{0}:\left(\vec{u}, \vec{u}_{1}, \sigma\right) \mapsto(\vec{u}, \hat{\sigma})$ maps any solution of Problem 4.1 to a solution of Problem 6.1, and the multivalued operator $R_{0}$ is its inverse. In the terminology of [40], $Q_{0}$ is a compression operator and $R_{0}$ is a (multivalued) reconstruction operator, This may be compared with the definitions (5.27), that concerned the constitutive relation. 
$\Gamma$-Convergence. The formulation of our evolution problem in terms of minimization suggests the possibility of retrieving the above homogenization result via De Giorgi's notion of $\Gamma$-convergence (see e.g. [17, 18,35,36]).

Let us remind the definitions (3.36) and (6.4) of the functionals $J_{0}$ and $J_{1}$. For any $\eta>0$ let us replace $x$ by $x / \eta$ in Problem 3.1, and use the index $\eta$ to label the corresponding functional and any minimizer of the associated functional $J_{0 \eta}$; that is,

$$
\begin{aligned}
J_{0 \eta}(\vec{u}, \sigma):= & \iint_{\Omega_{T}}\left[\varphi\left(\sigma, \varepsilon-A(x / \eta): \int_{0}^{t} \sigma, x / \eta\right)+\sigma: A(x / \eta): \int_{0}^{t} \sigma\right] d x d t \\
& -\int_{0}^{T}\langle\vec{f}, \vec{u}\rangle d t+I_{\mathcal{X}_{\vec{f}}}(\sigma) \quad \forall(\vec{u}, \sigma) \in L^{q}(0, T ; V) \times L^{p}\left(\Omega_{T}\right)_{s}^{9} .
\end{aligned}
$$

Theorem 6.4. Let the hypotheses of Theorem 4.4 be satisfied (so that the solution of Problem 6.1 exists and is unique). The family $\left\{J_{0 \eta}\right\}$ then $\Gamma$-converges weakly in $L^{q}(0, T ; V) \times L^{p}\left(\Omega_{T}\right)_{s}^{9}$ to $J_{1}$, that is, for any $(\vec{u}, \bar{\sigma}) \in L^{q}(0, T ; V) \times L^{p}\left(\Omega_{T}\right)_{s}^{9}$,

$$
\begin{aligned}
& \forall\left\{\left(\vec{u}_{\eta}, \sigma_{\eta}\right)\right\} \subset L^{q}(0, T ; V) \times L^{p}\left(\Omega_{T}\right)_{s}^{9}, \\
& \text { if } \vec{u}_{\eta} \rightarrow \vec{u} \text { in } L^{q}(0, T ; V) \text { and } \sigma_{\eta} \rightarrow \bar{\sigma} \text { in } L^{p}\left(\Omega_{T}\right)_{s}^{9}, \\
& \text { then } \liminf _{\eta \rightarrow 0} J_{0 \eta}\left(\vec{u}_{\eta}, \sigma_{\eta}\right) \geq J_{1}(\vec{u}, \bar{\sigma}), \\
& \exists\left\{\left(\vec{u}_{\eta}, \sigma_{\eta}\right)\right\} \subset L^{q}(0, T ; V) \times L^{p}\left(\Omega_{T}\right)_{s}^{9} \text { such that } \\
& \vec{u}_{\eta} \rightarrow \vec{u} \text { in } L^{q}(0, T ; V), \sigma_{\eta} \rightarrow \bar{\sigma} \text { in } L^{p}\left(\Omega_{T}\right)_{s}^{9} \text {, and } \\
& \lim \sup J_{0 \eta}\left(\vec{u}_{\eta}, \sigma_{\eta}\right) \leq J_{1}(\vec{u}, \bar{\sigma}) .
\end{aligned}
$$

Proof. (i) Let us first check (6.10) and (6.11) for $(\vec{u}, \bar{\sigma})$ equal to the solution of Problem 6.1. The inferior-limit property (6.10) easily follows from the convexity and lower semicontinuity of the function $\varphi$. For any $\eta$, let $\left(\vec{u}_{\eta}, \sigma_{\eta}\right)$ be the solution of Problem 3.1 $1_{\eta}$; as $J_{0 \eta}\left(\vec{u}_{\eta}, \sigma_{\eta}\right)=0$ and $J_{1}(\vec{u}, \sigma)=0$, the condition (6.11) is also fulfilled.

(ii) In view of proving the sequential weak $\Gamma$-convergence at any point

$$
(\overline{\vec{v}}, \bar{s}) \in \operatorname{Dom}\left(J_{1}\right)=L^{q}(0, T ; V) \times \mathcal{X}_{\vec{f}} \quad(\operatorname{see}(3.35)),
$$

next we linearly perturb the functionals $J_{0 \eta}$ and $J_{1}$, in such a way that $(\overline{\vec{v}}, \bar{s})$ will be a minimizer of the perturbed $J_{1}$. As $J_{1}$ is convex and finite on the whole affine subspace $L^{q}(0, T ; V) \times \mathcal{X}_{\vec{f}}$, and $L^{p}\left(0, T ; V^{\prime}\right) \times L^{q}\left(\Omega_{T}\right)_{s}^{9}$ is the dual of the ambient space $L^{q}(0, T ; V) \times L^{p}\left(\Omega_{T}\right)_{s}^{9}$,

$$
\emptyset \neq \partial J_{1}(\overline{\bar{v}}, \bar{s}) \subset \mathcal{P}\left(L^{p}\left(0, T ; V^{\prime}\right) \times L^{q}\left(\Omega_{T}\right)_{s}^{9}\right) \quad \forall(\overline{\bar{v}}, \bar{s}) \in L^{q}(0, T ; V) \times \mathcal{X}_{\vec{f}} .
$$


We then select any $\ell:=\left(\vec{\ell}_{1}, \ell_{2}\right) \in \partial J_{1}(\overline{\vec{v}}, \bar{s})$, and set

$$
\begin{gathered}
J_{0 \eta}^{(\ell)}(\vec{v}, s):=J_{0 \eta}(\vec{v}, s)-\int_{0}^{T}\left\langle\vec{\ell}_{1}, \vec{v}\right\rangle d t-\iint_{\Omega_{T}} \ell_{2}: s d x d t \\
J_{1}^{(\ell)}(\vec{v}, s):=J_{1}(\vec{v}, s)-\int_{0}^{T}\left\langle\vec{\ell}_{1}, \vec{v}\right\rangle d t-\iint_{\Omega_{T}} \ell_{2}: s d x d t \\
\forall(\vec{v}, s) \in L^{q}(0, T ; V) \times L^{p}\left(\Omega_{T}\right)_{s}^{9} .
\end{gathered}
$$

The properties of these linearly perturbed functionals are analogous to those of $J_{0 \eta}$ and $J_{1}$. By the definition of subdifferential, $J_{1}^{(\ell)}$ attains its minimum at $(\overline{\vec{v}}, \bar{s})$. By part (i) of this argument, $J_{0 \eta}^{(\ell)}$ then weakly $\Gamma$-converges to $J_{1}^{(\ell)}$ at $(\overline{\vec{v}}, \bar{s})$. This is tantamount to the weak $\Gamma$-convergence of $J_{0 \eta}$ to $J_{1}$ at the same point. As $(\overline{\vec{v}}, \bar{s})$ is just any point of the domain of $J_{1}$, the weak $\Gamma$-convergence of $J_{0 \eta}$ to $J_{1}$ in the whole space $L^{q}(0, T ; V) \times L^{p}\left(\Omega_{T}\right)_{S}^{9}$ is thus established.

Analogical models. Several rheological laws, including (1.1) and (1.8), may be represented via so-called analogical models, namely networks of elements that are arranged in series and/or in parallel. Similar procedures are used in electrostatics, in magnetostatics, in electromagnetism, and so on, see e.g. $[1,2,43,49,56,76]$. Their significance is not yet clear, and seems to be essentially heuristic.

Let us combine in series a finite family $\left\{M_{j}: j=1, \ldots, N\right\}$ of univariate models like (1.1), each one being characterized by two functions $\alpha_{j}(\cdot, x)$ and $A_{j}(x)$. Each element $M_{j}$ defines a mapping $\mathcal{F}_{j}: \varepsilon_{j} \mapsto \sigma_{j}$ for any $j$. This arrangement is associated to the following properties:

(i) mean-field hypothesis: $\varepsilon_{j}$ is independent of $j$ and equals the strain $\varepsilon$ of the overall model;

(ii) additivity: $\sigma=\sum_{j=1}^{N} \sigma_{j}$, that is,

$$
\sigma=\sum_{j=1}^{N} \mathcal{F}_{j}\left(\varepsilon_{j}\right)=\sum_{j=1}^{N} \mathcal{F}_{j}(\varepsilon)=: \hat{\mathcal{F}}(\varepsilon)
$$

Finite parallel arrangements are governed by the dual properties: $\sigma_{j}$ is independent of $j$, and the overall $\varepsilon$ equals the sum of the $\varepsilon j$ 's. Both constructions take over to a countable family of elements just by replacing finite sums by series. In the continuous setting the index $j$ may be replaced by the fine-scale variable $y \in \mathcal{Y}$, and sums by integrals over the reference cell $\mathcal{Y}$. A large class of univariate models can be constructed by this procedure, and these constitutive relations take over to the multivariate setting just by extending the properties (i) and (ii). In the latter case the interpretation in terms of series and parallel arrangements obviously fails.

A comparison between the outcome of this construction and the above twoand single-scale models seems natural. In general the constitutive relations that is derived via homogenization need not be equivalent to those issued from analogical models, for the latter rest on the (unjustified) a priori assumption that either the 
stress or the strain should be mesoscopically uniform, viz. independent of the finescale variable $y$. This uniformity would hold if the solution $\left(\vec{u}_{\eta}, \sigma_{\eta}\right)$ of Problems $3.1_{\eta}$ were such that either the family $\left\{\varepsilon_{\eta}:=\nabla \vec{u}_{\eta}: \eta>0\right\}$ or $\left\{\sigma_{\eta}: \eta>0\right\}$ satisfy a uniform estimate in a Sobolev space $W^{r, p}(\mathcal{Y})_{s}^{9}$ for some $r>0$ and $p \geq 1$. However, no estimate like that seems available, although $-\nabla \cdot \sigma_{\eta}=\vec{f}$, and the symmetrized gradient $\varepsilon_{\eta}:=\nabla^{s} \vec{u}$ fulfills a (second-order) compatibility equation. One may thus expect that in general the material will exhibit a nontrivial mesoscopic structure, that cannot be integrated simply by assuming either the above properties (i) and (ii), or their dual formulation for parallel arrangements.

\section{References}

[1] H.-D. ALBER, Global existence and boundedness of large solutions to nonlinear equations of viscoelasticity with hardening, Commun. Math. Phys. 166 (1995), 565-601.

[2] H.-D. AlBeR, "Materials with Memory", Springer, Berlin 1998.

[3] H.-D. ALBER, Justification of homogenized models for viscoplastic bodies with microstructure, In: "Deformation and Failure in Metallic Materials", K. Hutter and H. Baaser (eds.), Springer, Berlin, 2003, 295-319.

[4] G. Allaire, Homogenization and two-scale convergence, SIAM J. Math. Anal. 23 (1992), $1482-1518$.

[5] G. Allaire, "Shape Optimization by the Homogenization Method", Springer, New York, 2002.

[6] S. S. Antman, "Nonlinear Problems of Elasticity", Springer, New York, 2005.

[7] T. Arbogast, J. Douglas and U. Hornung, Derivation of the double porosity model of single phase flow via homogenization theory, SIAM J. Math. Anal. 21 (1990), 823-836.

[8] J.-P. AUBIN and I. EKELAND, Second-order evolution equations associated with convex Hamiltonians, Canad. Math. Bull. 23 (1980), 81-94.

[9] G. AuCHMUTY, Saddle-points and existence-uniqueness for evolution equations, Differential Integral Equations 6 (1993), 1161-1171.

[10] I. BABUŠKA, Homogenization and its application. Mathematical and computational problems, In: "Numerical Solution of Partial Differential Equations III" (College Park, Md., 1975), Academic Press, New York, 1976, 89-116.

[11] M. BAÍA and I. FONSECA, The limit behavior of a family of variational multiscale problems, Indiana Univ. Math. J. 56 (2007), 1-50.

[12] N. S. BAKhVAlov and G. P. PAnasenko, "Homogenisation: Averaging Processes in Periodic Media. Mathematical Problems in the Mechanics of Composite Materials", Kluwer, Dordrecht, 1989.

[13] J. M. BALL, Convexity conditions and existence theorems in nonlinear elasticity, Arch. Ration. Mech. Anal. 63 (1976/77), 337-403.

[14] G. Bensoussan, J. L. Lions and G. Papanicolaou, "Asymptotic Analysis for Periodic Structures", North-Holland, Amsterdam, 1978.

[15] D. BlanCHARD and P. LE TALlEC, Numerical analysis of the equations of small strains quasistatic elastoviscoplasticity, Numer. Math. 50 (1986), 147-169.

[16] D. Blanchard, P. Le TAllec and M. Ravachol, Numerical analysis of evolution problems in nonlinear small strains elastoviscoplasticity, Numer. Math. 55 (1989), 177195.

[17] A. BRAides, " $\Gamma$-Convergence for Beginners", Oxford University Press, Oxford, 2002.

[18] A. Braides and A. Defranceschi, "Homogenization of Multiple Integrals", Oxford University Press, Oxford, 1998. 
[19] H. BRezIs, "Opérateurs Maximaux Monotones et Semi-Groupes de Contractions dans les Espaces de Hilbert", North-Holland, Amsterdam, 1973.

[20] H. BREZIS and I. EKELAND, Un principe variationnel associé à certaines équations paraboliques. I.Le cas indépendant du temps, and II.Le cas dépendant du temps, C. R. Acad. Sci. Paris Sér. A-B 282 (1976), 971-974, and ibid. 1197-1198.

[21] F. BROWDER, Existence theorems for nonlinear partial differential equations, In: "Proceedings of Symposia in Pure Mathematics", Vol. XVI, S. Chern and S. Smale (eds.) AMS, Providence (1970), 1-60.

[22] R. S. BURACHIK and B. F. SVAITER, Maximal monotone operators, convex functions and a special family of enlargements, Set-Valued Anal. 10 (2002), 297-316.

[23] R. S. BURACHIK and B. F. SVAITER, Maximal monotonicity, conjugation and the duality product, Proc. Amer. Math. Soc. 131 (2003), 2379-2383.

[24] L. CARBONE and C. SBORDONE, Some properties of $\Gamma$-limits of integral functionals, Ann. Mat. Pura Appl. (4) 122 (1979), 1-60.

[25] A. Cherkaev and R. Kohn (eds.), "Topics in the Mathematical Modelling of Composite Materials", Birkhäuser, Boston, 1997.

[26] V. ChIADÒ PIAT and G. V. SANDRAKOV, Homogenization of some variational inequalities for elasto-plastic torsion problems, Asymptot. Anal. 40 (2004), 1-23.

[27] R. M. Christensen, "Theory of Viscoelasticity”, Academic Press, New York, 1971.

[28] Ph. Ciarlet, "Mathematical Elasticity, Vol. I: Three-Dimensional Elasticity", NorthHolland, Amsterdam, 1988.

[29] D. Cioranescu, A. Damlamian and R. De ArCangelis, Homogenization of nonlinear integrals via the periodic unfolding method, C.R. Acad. Sci. Paris, Ser. I 339 (2004), $77-82$.

[30] D. Cioranescu, A. Damlamian and R. De ArCangelis, Homogenization of quasiconvex integrals via the periodic unfolding method, SIAM J. Math. Anal. 37 (2006), 14351453.

[31] D. Cioranescu, A. Damlamian and G. Griso, Periodic unfolding and homogenization, C.R. Acad. Sci. Paris, Ser. I 335 (2002), 99-104.

[32] D. Cioranescu, A. Damlamian and G. Griso, The periodic unfolding method in homogenization., SIAM J. Math. Anal. 40 (2008), 1585-1620.

[33] D. Cioranescu and P. Donato, "An Introduction to Homogenization", Oxford Univ. Press, New York, 1999.

[34] B. Dacorogna, "Direct Methods in the Calculus of Variations", Springer, Berlin, 1989.

[35] G. DAL Maso, "An Introduction to $\Gamma$-Convergence", Birkhäuser, Boston, 1993.

[36] E. De Giorgi and T. Franzoni, Su un tipo di convergenza variazionale, Atti Accad. Naz. Lincei Rend. Cl. Sci. Fis. Mat. Natur. (8) 58 (1975), 842-850.

[37] E. De GioRgi and S. SPAgnolo, Sulla convergenza degli integrali dell'energia per operatori ellittici del secondo ordine, Boll. Un. Mat. Ital. 8 (1973), 391-411.

[38] G. Duvaut and J. L. Lions, "Les Inéquations en Mécanique et en Physique", Dunod, Paris, 1972.

[39] I. Ekeland and R. Temam, "Analyse Convexe et Problèmes Variationnelles", Dunod Gauthier-Villars, Paris, 1974.

[40] W. E. B. EngQuist, The heterogeneous multiscale methods, Commun. Math. Sci. 1 (2003), 87-132.

[41] W. FENCHEL, "Convex Cones, Sets, and Functions", Princeton Univ., 1953.

[42] S. FitZPATRICK, Representing monotone operators by convex functions, In: "Workshop/Miniconference on Functional Analysis and Optimization" (Canberra, 1988), Proc. Centre Math. Anal. Austral. Nat. Univ., Vol. 20, Austral. Nat. Univ., Canberra, 1988, 59-65.

[43] W. FLÜGGE, "Viscoelasticity", Springer, Berlin, 1975.

[44] G. Francfort, D. LEguILlON and P. SUQUeT, Homogénéisation de milieux viscoélastiques linéaires de Kelvin-Voigt, C. R. Acad. Sci. Paris Sér. I Math. 296 (1983), 287-290. 
[45] G. FRANCFORT and P. SUQUeT, Homogenization and mechanical dissipation in thermoviscoelasticity, Arch. Rational Mech. Anal. 96 (1986), 265-293.

[46] G. GeYmonat, P. SuQuet, Functional spaces for Norton-Hoff materials, Math. Methods Appl. Sci. 8 (1986), 206-222.

[47] N. Ghoussoub, "Self-dual Partial Differential Systems and their Variational Principles", Springer, 2009.

[48] M.E. GurTin, The linear theory of elasticity, In: "Handbuch der Physik", S. Flügge (ed.), Vol. VIa/2. Springer, Berlin, 1972, 1-295.

[49] B. Halphen and NguYen Quoc Son, Sur les matériaux standard généralisés, J. Méchanique 14 (1975), 39-63.

[50] W. Han, B.D. RedDY, "Plasticity", Springer, New York, 1999.

[51] J.-B. Hiriart-UrRuty and C. Lemarechal, "Convex Analysis and Optimization Algorithms", Springer, Berlin, 1993.

[52] U. HoRnung (ed.), "Homogenization and Porous Media", Springer, New York, 1997.

[53] A.D. IoffE and V. M. Tinomirov, "Theory of Extremal Problems", North-Holland, Amsterdam, 1979.

[54] V. V. JiKov, S. M. Kozlov and O. A. Oleinik, "Homogenization of Differential Operators and Integral Functionals", Springer, Berlin, 1994.

[55] M. J. LeITMAN and G. M. C. FISHER, The linear theory of viscoelasticity. In: "Handbuch der Physik", S. Flügge (ed.), Vol. VIa/3. Springer, Berlin 1973, 1-123.

[56] J. Lemaitre and J.-L. Chaboche, "Mechanics of Solid Materials", Cambridge Univ. Press, Cambridge, 1990.

[57] P. LE TALlEC, "Numerical Analysis of Viscoelastic Problems", Masson and Springer, Paris, 1990.

[58] J. L. LiONS, "Quelques méthodes de résolution des problèmes aux limites non linéaires", Dunod, Paris, 1969.

[59] D. LukKassen, G. Nguetseng and P. Wall, Two-scale convergence, Int. J. Pure Appl. Math. 2 (2002), 35-86.

[60] P. Marcellini, Periodic solutions and homogenization of nonlinear variational problems, Ann. Mat. Pura Appl. (4) 117 (1978), 139-152.

[61] J.-E. MARTINEZ-LEGAZ and B. F. SVAITER, Monotone operators representable by l.s.c. convex functions, Set-Valued Anal. 13 (2005), 21-46.

[62] J.-E. MartineZ-LEgAZ and B. F. SVAITER, Minimal convex functions bounded below by the duality product, Proc. Amer. Math. Soc. 136 (2008), 873-878.

[63] J.-E. MARTINEZ-LEGAZ and M. THÉRA, A convex representation of maximal monotone operators, J. Nonlinear Convex Anal. 2 (2001), 243-247.

[64] G. A. Maugin, "The Thermodynamics of Plasticity and Fracture", Cambridge Univ. Press, Cambridge, 1992.

[65] A. Mielke and A. TIMofTe, Two-scale homogenization for evolutionary variational inequalities via the energetic formulation, SIAM J. Math. Anal. 39 (2007), 642-668.

[66] G. W. Milton, “The Theory of Composites”, Cambridge Univ. Press, Cambridge, 2002.

[67] J. J. Moreau, Fonctionnelles Convexes, Séminaires sur les équations aux derivées partielles, Collége de France, Paris, 1967.

[68] F. Murat, Compacité par compensation, Ann. Scuola Norm. Sup. Pisa (4) 5 (1978), 489507.

[69] F. MURAT and L. TARTAR, H-convergence, In: "Topics in the Mathemtical Modelling of Composite Materials", Progr. Nonlinear Differential Equations Appl., Vol. 31, Birkhäuser, Boston, 1997, 21-43.

[70] B. NAYRoles, Deux théorèmes de minimum pour certains systèmes dissipatifs, C. R. Acad. Sci. Paris Sér. A-B 282 (1976), A1035-A1038.

[71] J. NEČAS and I. HLAVÁČEK, "Mathematical Theory of Elastic and Elastico-Plastic Bodies: an Introduction", Elsevier, Amsterdam, 1982. 
[72] S. Nesenenko, Homogenization in viscoplasticity, SIAM J. Math. Anal. 39 (2007), 236262.

[73] G. NGuetseng, A general convergence result for a functional related to the theory of homogenization, SIAM J. Math. Anal. 20 (1989), 608-623.

[74] O. A. Oleinik, A. S. Shamaev and G. A. Yosifian, "Mathematical Problems in Elasticity and Homogenization", North-Holland, Amsterdam, 1992.

[75] A. PANKOV, " $G$-Convergence and Homogenization of Nonlinear Partial Differential Operators", Kluwer, Dordrecht, 1997.

[76] M. ReIneR, Rheology, In: "Handbuch der Physik", S. Flügge (ed.), Vol. VI. Springer, Berlin, 1958, 434-550.

[77] M. Renardy, W. J. Hrusa and J. A. Nohel, "Mathematical Problems in Viscoelasticity", Longman Scientific \& Technical, Harlow; John Wiley \& Sons, New York, 1987.

[78] R.T. Rock afellar, "Convex Analysis", Princeton University Press, Princeton, 1969.

[79] E. SANChEZ-PALENCIA, "Non-Homogeneous Media and Vibration Theory", Springer, New York, 1980.

[80] R. E. ShOWALTER, "Monotone Operators in Banach Spaces and Nonlinear Partial Differential Equations", Mathematical Surveys and Monographs, Vol. 49, American Mathematical Society, Providence, RI, 1997.

[81] S. Spagnolo, Sulla convergenza di soluzioni di equazioni paraboliche ed ellittiche, Ann. Scuola Norm. Sup. Pisa (3) 22 (1968), 571-597; errata, ibid. (3) 22 (1968), 673.

[82] L. TARTAR, Course Peccot, Collège de France, Paris 1977. (Unpublished, partially written in [25])

[83] L. TARTAR, Compensated compactness and applications to partial differential equations, In: "Nonlinear Analysis and Mechanics: Heriott-Watt Symposium", Vol. IV, R. J. Knops (ed.), Pitman, London 1979, 136-212.

[84] R. TEMAM, "Problèmes Mathématiques en Plasticité", Gauthier-Villars, Paris, 1983.

[85] A. Visintin, Homogenization of the nonlinear Kelvin-Voigt model of visco-elasticity and of the Prager model of plasticity, Continuum Mech. Thermodyn. 18 (2006), 223-252.

[86] A. Visintin, Two-scale convergence of first-order operators, Z. Anal. Anwendungen 26 (2007), 133-164.

[87] A. Visintin, Homogenization of the nonlinear Maxwell model of viscoelasticity and of the Prandtl-Reuss model of elastoplasticity, Royal Soc. Edinburgh Proc. A 138 (2008), 1-39.

[88] A. Visintin, Two-scale convergence of some integral functionals, Calc. Var. Partial Differential Equations 29 (2007), 239-265.

[89] A. Visintin, Homogenization of nonlinear visco-elastic composites, J. Math. Pures Appl. 89 (2008), 477-504.

[90] A. VISINTIN, Scale-transformations and homogenization of maximal monotone relations, with applications, forthcoming.

[91] E. ZEIDLER, "Nonlinear Functional Analysis and its Applications", Vol. II. Springer, New York, 1985.

Università degli Studi di Trento Dipartimento di Matematica via Sommarive 14 38050 Povo (Trento), Italia Visintin@science.unitn.it 